\title{
Direct adaptive command following and disturbance rejection for minimum phase systems with unknown relative degree
}

\author{
Jesse B. Hoagg*,† and Dennis S. Bernstein \\ Department of Aerospace Engineering, The University of Michigan, Ann Arbor, MI 48109-2140, U.S.A.
}

\begin{abstract}
SUMMARY
This paper considers parameter-monotonic direct adaptive command following and disturbance rejection for single-input single-output minimum-phase linear time-invariant systems with knowledge of the sign of the high-frequency gain (first non-zero Markov parameter) and an upper bound on the magnitude of the high-frequency gain. We assume that the command and disturbance signals are generated by a linear system with known characteristic polynomial. Furthermore, we assume that the command signal is measured, but the disturbance signal is unmeasured. The first part of the paper is devoted to a fixed-gain analysis of a high-gain-stabilizing dynamic compensator for command following and disturbance rejection. The compensator utilizes a Fibonacci series construction to control systems with unknown-but-bounded relative degree. We then introduce a parameter-monotonic adaptive law and guarantee asymptotic command following and disturbance rejection. Copyright (C) 2006 John Wiley \& Sons, Ltd.
\end{abstract}

Received 19 August 2005; Revised 6 September 2006; Accepted 25 September 2006

KEY WORDS: adaptive; relative degree; command following; disturbance rejection

\section{INTRODUCTION}

Direct adaptive stabilization methods include direct model reference adaptive control, high-gain adaptive control, and universal stabilization. Direct model reference adaptive controllers rely on parameter estimation algorithms such as recursive least-squares, gradient descent, and projection algorithms to update the controller parameters [1-3]. These adaptation schemes generally result in adaptive controllers whose dimension increases at least linearly or even quadratically with the order of the plant.

Alternatively, high-gain adaptive stabilization methods use simple adaptation laws and rely on a minimum-phase assumption since zeros attract poles under high gain [4-14]. Adaptive high-gain proportional feedback can stabilize square multi-input, multi-output systems that are minimum phase and relative degree one $[4,6,7]$.

*Correspondence to: Jesse B. Hoagg, Department of Aerospace Engineering, The University of Michigan, Ann Arbor, MI 48109-2140, U.S.A.

†E-mail: jhoagg@umich.edu 
Generally, high-gain methods can stabilize systems with relative degree one. However, in [8], high-gain dynamic compensation is used to guarantee output convergence of single-input singleoutput (SISO) minimum-phase systems with arbitrary-but-known relative degree. This result is surprising since classical roots locus is not high-gain stable for plants with relative degree exceeding two. However, in $[13,14]$ it is shown that the results of [8] can fail when the relative degree of the plant exceeds four. Furthermore, in $[13,14]$, the Fibonacci series is used to construct a direct adaptive stabilization algorithm for minimum-phase systems with unknownbut-bounded relative degree.

In general, high-gain adaptive methods are restricted to the stabilization problem. However, high-gain adaptive controllers that utilize high-gain observers have been used for $\lambda$-tracking $[15,16]$. The adaptive command following problem is usually addressed using model reference adaptive control. Direct model reference adaptive controllers cancel the zeros of the plant and replace them with the zeros of the reference model, thus requiring that the plant be minimum phase. In addition, direct model reference adaptive controllers typically require knowledge of the relative degree and a bound on the plant order. The bound on the plant order is used to determine the order of the model reference controller, while knowledge of the relative degree is required for developing the adaptation law [2]. Model reference adaptive control methods with relaxed assumptions on the relative degree of the plant are considered in [17, 18]. Specifically, in [17], a model reference adaptive controller is proposed for plants with relative degree one or two. However, the method is restricted to stabilization and does not address command following. In [18], a model reference adaptive controller is proposed for systems with upper and lower bounded relative degree. This controller requires that an adaptive parameter lie inside a known convex set, and for large uncertainty in relative degree, calculating the convex set can be difficult.

In the present paper, we extend the Fibonacci-based adaptive stabilization controller presented in $[13,14]$ to address the adaptive command following and disturbance rejection problems. We assume that the command and disturbance signals are generated by a linear system with known characteristic polynomial. However, the disturbance is unmeasured. Unlike direct model reference adaptive controllers, this adaptive controller does not require a bound on plant order or knowledge of the relative degree. Additionally, the method presented in this paper simultaneously addresses the command following and disturbance rejection problem, whereas model reference adaptive control is generally restricted to the command following problem. The main result of this paper is direct adaptive command following and disturbance rejection for SISO minimum-phase systems with unknown-but-bounded relative degree.

In Section 2, we present the SISO command following and disturbance rejection problem and introduce the notion of parameter-dependent dynamic compensation. Section 3 presents a fixed-gain analysis of Fibonacci-based high-gain stabilization. In Section 4, the Fibonacci-based compensator is extended to address the command following and disturbance rejection problem. The main result is given in Section 5. Numerical examples are given in Section 6 and conclusions in Section 7.

\section{PARAMETER-DEPENDENT DYNAMIC COMPENSATION FOR COMMAND FOLLOWING AND DISTURBANCE REJECTION}

We consider the strictly proper SISO linear time-invariant system

$$
y=G(s)(u+w)
$$

Copyright (C) 2006 John Wiley \& Sons, Ltd.

Int. J. Adapt. Control Signal Process. 2007; 21:49-75

DOI: $10.1002 /$ acs 
where

$$
G(s) \triangleq \delta \beta \frac{z(s)}{p(s)}
$$

$z(s)$ and $p(s)$ are real monic polynomials, $\delta= \pm 1$ is the sign of the high-frequency gain, and $\beta>0$ is the magnitude of the high-frequency gain. We define the notation $m \triangleq \operatorname{deg} z(s)$, $n \triangleq \operatorname{deg} p(s)$, and $r \triangleq n-m$. Furthermore, we consider a command signal $y_{\mathrm{r}}(t)$ and a disturbance signal $w(t)$ that are generated by the exogenous dynamics

$$
\dot{x}_{\mathrm{r}}(t)=A_{\mathrm{r}} x_{\mathrm{r}}(t), \quad u_{\mathrm{r}}(t)=C_{\mathrm{r}} x_{\mathrm{r}}(t)
$$

where $u_{\mathrm{r}}(t) \triangleq\left[y_{\mathrm{r}}(t) w(t)\right]^{\mathrm{T}}, A_{\mathrm{r}} \in \mathbb{R}^{n_{\mathrm{r}} \times n_{\mathrm{r}}}, C_{\mathrm{r}} \in \mathbb{R}^{2 \times n_{\mathrm{r}}}$, and the characteristic polynomial of $A_{\mathrm{r}}$ is $p_{\mathrm{r}}(s) \triangleq \operatorname{det}\left(s I-A_{\mathrm{r}}\right)$.

In this paper, we address the adaptive command following and disturbance rejection problem for system (1) and (2). The objective is to construct an adaptive controller that forces the plant output $y$ to asymptotically follow the command signal $y_{\mathrm{r}}$ while rejecting the unmeasured disturbance $w$. We make the following assumptions.

(I) $z(s)$ is a real monic Hurwitz polynomial but is otherwise unknown.

(II) $p(s)$ is a real monic polynomial but is otherwise unknown.

(III) $z(s)$ and $p(s)$ are coprime.

(IV) The magnitude $\beta$ of the high-frequency gain satisfies $0<\beta \leqslant b_{0}$, where $b_{0} \in \mathbb{R}$ is known.

(V) The sign $\delta= \pm 1$ of the high-frequency gain is known.

(VI) The relative degree $r$ of $G(s)$ satisfies $1 \leqslant r \leqslant \rho$, where $\rho$ is known, but $r$ is otherwise unknown.

(VII) For all $\lambda \in \operatorname{spec}\left(A_{\mathrm{r}}\right), \operatorname{Re} \lambda=0$ and $\lambda$ is semisimple.

(VIII) The command signal $y_{\mathrm{r}}$ is measured.

(IX) The disturbance signal $w$ is not measured.

(X) The characteristic polynomial $p_{\mathrm{r}}(s)$ is known.

Assumption (VII) restricts our attention to command and disturbance signals that consist of steps and sinusoids. Furthermore, assumption (X) implies that the command and disturbance sinusoids have known frequencies. However, assumption (IX) implies that we do not require a direct measurement of the disturbance.

Let $c_{k}(s)$ and $d_{k}(s)$ be parameter-dependent polynomials, that is, polynomials in $s$ over the reals whose coefficients are functions of a real parameter $k$. Furthermore, define the parameterdependent transfer function

$$
H_{k}(s) \triangleq \frac{c_{k}(s)}{d_{k}(s)}
$$

where, for all $k \in \mathbb{R}, p_{k}(\cdot) \not \equiv 0$. Note that the polynomials $c_{k}(s)$ and $d_{k}(s)$ need not be coprime for all $k \in \mathbb{R}$.

\section{Definition 2.1}

$d_{k}(s)$ is high-gain Hurwitz if there exists $k_{\mathrm{s}}>0$ such that $d_{k}(s)$ is Hurwitz for all $k \geqslant k_{\mathrm{s}}$.

Definition 2.2

$H_{k}(s)$ is high-gain stable if there exist parameter-dependent polynomials $c_{k}(s)$ and $d_{k}(s)$ such that $d_{k}(s)$ is high-gain Hurwitz and, for all $k \in \mathbb{R}, G_{k}(s)=c_{k}(s) / d_{k}(s)$. 
Now, consider the feedback controller

$$
u=\hat{G}_{k}(s) y_{e}
$$

where

$$
\hat{G}_{k}(s) \triangleq \frac{\hat{z}_{k}(s)}{\hat{p}_{k}(s)}
$$

$y_{e} \triangleq y_{\mathrm{r}}-y$ is the output error, and $\hat{z}_{k}(s)$ and $\hat{p}_{k}(s)$ are parameter-dependent polynomials. For example, letting $\hat{z}_{k}(s)=\delta k$ and $\hat{p}_{k}(s)=1$ yields $\hat{G}_{k}(s)=\delta k$, and the closed-loop poles can be determined by classical root locus. In general, the closed-loop system (1), (2), (4), and (5) from the command $y_{\mathrm{r}}$ and disturbance $w$ to the tracking error $y_{e}$ is

$$
y_{e}=\tilde{G}_{k}(s) u_{\mathrm{r}}=\left[\begin{array}{ll}
\tilde{G}_{k, 1}(s) & \tilde{G}_{k, 2}(s)
\end{array}\right]\left[\begin{array}{c}
y_{\mathrm{r}} \\
w
\end{array}\right]
$$

where

$$
\begin{aligned}
& \tilde{G}_{k, 1}(s) \triangleq \frac{1}{1+G(s) \hat{G}_{k}(s)}=\frac{\tilde{z}_{k, 1}(s)}{\tilde{p}_{k}(s)} \\
& \tilde{G}_{k, 2}(s) \triangleq \frac{-G(s)}{1+G(s) \hat{G}_{k}(s)}=\frac{\tilde{z}_{k, 2}(s)}{\tilde{p}_{k}(s)}
\end{aligned}
$$

and

$$
\begin{aligned}
\tilde{z}_{k, 1}(s) & \triangleq p(s) \hat{p}_{k}(s) \\
\tilde{z}_{k, 2}(s) & \triangleq-\delta \beta z(s) \hat{p}_{k}(s) \\
\tilde{p}_{k}(s) & \triangleq p(s) \hat{p}_{k}(s)+\delta \beta z(s) \hat{z}_{k}(s)
\end{aligned}
$$

The parameter-dependent closed-loop transfer functions $\tilde{G}_{k, 1}(s)$ and $\tilde{G}_{k, 2}(s)$ are high-gain stable if the parameter-dependent characteristic polynomial $\tilde{p}_{k}(s)$ is high-gain Hurwitz. The SISO command following and disturbance rejection problem is shown in Figure 1.

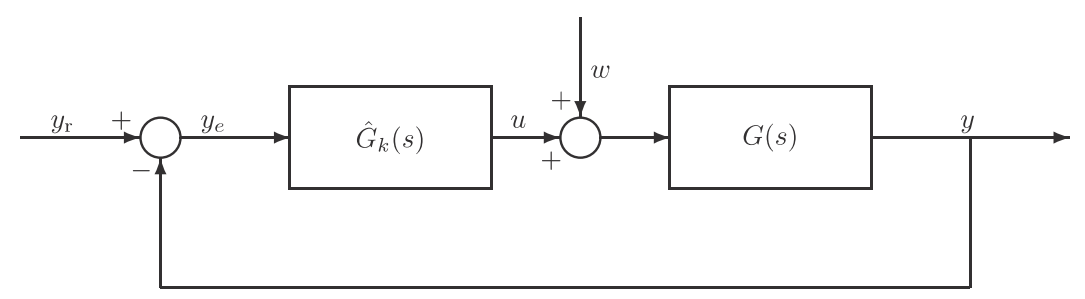

Figure 1. Combined command following and disturbance rejection problem. 


\section{HIGH-GAIN DYNAMIC COMPENSATION FOR STABILIZATION}

In this section, a parameter-dependent dynamic compensator is used to high-gain stabilize (1) and (2). The controller construction utilizes the Fibonacci series. For all $j \geqslant 0$ let $F_{j}$ be the $j$ th Fibonacci number, where $F_{0}=0, F_{1}=1, F_{2}=1, F_{3}=2, F_{4}=3, F_{5}=5, F_{6}=8, F_{7}=13$, $F_{8}=21, \ldots$, and define

$$
f_{g, h} \triangleq F_{g+2}-F_{h+1}
$$

where $h$ satisfies $1 \leqslant h \leqslant g$.

Consider the parameter-dependent dynamic compensator

$$
\hat{G}_{k, g}(s) \triangleq \frac{\delta k^{F_{g+2}} \hat{z}(s)}{s^{g}+k^{f_{g, g}} b_{g} s^{g-1}+k^{f_{g, g-1}} b_{g-1} s^{g-2}+\cdots+k^{f_{g, 2}} b_{2} s+k^{f_{g, 1}} b_{1}}
$$

where $k, b_{1}, \ldots, b_{g}$ are real, and $\hat{z}(s)$ is a degree $g-1$ monic polynomial.

Now, let $g$ be the upper bound on the relative degree of $G(s)$, that is, $g=\rho$, and consider the feedback (4) with $\hat{G}_{k}(s)=\hat{G}_{k, \rho}(s)$. Then closed-loop system (1), (2), (4), and (6) is

$$
y_{e}=\left[\begin{array}{ll}
\tilde{G}_{k, 1}(s) & \tilde{G}_{k, 2}(s)
\end{array}\right]\left[\begin{array}{l}
y_{\mathrm{r}} \\
w
\end{array}\right]
$$

where

$$
\begin{gathered}
\tilde{G}_{k, 1}(s) \triangleq \frac{1}{1+G(s) \hat{G}_{k}(s)}=\frac{\tilde{z}_{k, 1}(s)}{\tilde{p}_{k}(s)} \\
\tilde{G}_{k, 2}(s) \triangleq \frac{-G(s)}{1+G(s) \hat{G}_{k}(s)}=\frac{\tilde{z}_{k, 2}(s)}{\tilde{p}_{k}(s)}
\end{gathered}
$$

and

$$
\begin{gathered}
\tilde{z}_{k, 1}(s) \triangleq p(s)\left[s^{\rho}+k^{f_{\rho, \rho}} b_{\rho} s^{\rho-1}+k^{f_{\rho, \rho-1}} b_{\rho-1} s^{\rho-2}+\cdots+k^{f_{\rho, 2}} b_{2} s+k^{f_{\rho, 1}} b_{1}\right] \\
\tilde{z}_{k, 2}(s) \triangleq-\delta \beta z(s)\left[s^{\rho}+k^{f_{\rho, \rho}} b_{\rho} s^{\rho-1}+k^{f_{\rho, \rho-1}} b_{\rho-1} s^{\rho-2}+\cdots+k^{f_{\rho, 2}} b_{2} s+k^{f_{\rho, 1}} b_{1}\right] \\
\tilde{p}_{k}(s) \triangleq p(s) s^{\rho}+k^{f_{\rho, \rho}} b_{\rho} p(s) s^{\rho-1}+k^{f_{\rho, \rho-1}} b_{\rho-1} p(s) s^{\rho-2}+\cdots+k^{f_{\rho, 1}} b_{1} p(s)+k^{F_{\rho+2}} \beta z(s) \hat{z}(s)
\end{gathered}
$$

The following theorem provides the properties of $\tilde{p}_{k}(s)$, and thus $\tilde{G}_{k, 1}(s)$ and $\tilde{G}_{k, 2}(s)$ for sufficiently large $k$. The proof follows from examining the Hurwitz conditions of $\tilde{p}_{k}(s)$ for large $k$. For a complete proof of this result, see [13,14].

\section{Theorem 3.1}

Consider the closed-loop system (7)-(12). Assume that the polynomials $\hat{z}(s)$,

$$
B_{\rho-2}(s) \triangleq s^{3}+b_{\rho} s^{2}+b_{\rho-1} s+b_{0}
$$

and, for $i=0,1, \ldots, \rho-3$,

$$
B_{i}(s) \triangleq b_{i+3} s^{3}+b_{i+2} s^{2}+b_{i+1} s+b_{0}
$$


are Hurwitz. Then $\tilde{p}_{k}(s)$ is high-gain Hurwitz, and thus $\tilde{G}_{k, 1}(s)$ and $\tilde{G}_{k, 2}(s)$ are high-gain stable. Furthermore, as $k \rightarrow \infty, m+\rho-1$ roots of $\tilde{p}_{k}(s)$ converge to the roots of $z(s) \hat{z}(s)$ and the real parts of the remaining $r+1$ roots approach $-\infty$.

Note that there exist controller parameters $b_{1}, \ldots, b_{\rho}$ such that the polynomials $B_{0}(s), \ldots$, $B_{\rho-2}(s)$ are Hurwitz. First, let $b_{\rho}>0$ and $b_{\rho-1}>0$ be such that $b_{\rho-1} b_{\rho}>b_{0}$, which implies that $B_{\rho-2}(s)$ is Hurwitz. Next, let $b_{\rho-2}>b_{0} b_{\rho} / b_{\rho-1}$, which implies that $B_{\rho-3}(s)$ is Hurwitz. In the same manner, for $i=4,5, \ldots, \rho$, let $b_{\rho-i+1}>b_{0} b_{\rho-i+3} / b_{\rho-i+2}$ so that $B_{\rho-i}(s)$ is Hurwitz. Thus $B_{0}(s), \ldots, B_{\rho-2}$ are Hurwitz.

The parameter-dependent dynamic compensator $\hat{G}_{k, \rho}(s)$ is high-gain stabilizing for $G(s)$ under assumptions (I)-(X). However, the closed-loop system (7)-(12) is not guaranteed to asymptotically follow the command signal or reject the disturbance. In fact, the closed-loop system will not generally follow the command signal or reject the disturbance since $\hat{G}_{k, \rho}(s)$ does not have an internal model of $p_{\mathrm{r}}(s)$ for all values of $k$. However, in the next section, we augment $\hat{G}_{k, \rho}(s)$ to incorporate an internal model of $p_{\mathrm{r}}(s)$.

\section{HIGH-GAIN DYNAMIC COMPENSATION FOR COMMAND FOLLOWING AND DISTURBANCE REJECTION}

In this section, we construct a high-gain dynamic compensator for command following and disturbance rejection by cascading an internal model of the exogenous dynamics $p_{\mathrm{r}}(s)$ with $\hat{G}_{k, g}(s)$, where the parameter $g$ is chosen to be an upper bound on the relative degree of an augmented system.

Consider the feedback (4) with the strictly proper dynamic compensator

$$
\hat{G}_{k}(s) \triangleq \hat{G}_{\mathrm{r}}(s) \hat{G}_{k, \bar{\rho}}(s)
$$

where $\hat{G}_{\mathrm{r}}(s) \triangleq \hat{z}_{\mathrm{r}}(s) / p_{\mathrm{r}}(s), \hat{z}_{\mathrm{r}}(s)$ is a monic polynomial with $m_{\mathrm{r}} \triangleq \operatorname{deg} \hat{z}_{\mathrm{r}}(s) \leqslant n_{\mathrm{r}}$, and $\hat{G}_{k, \bar{\rho}}(s)$ is given by (6) with $g=\bar{\rho}$, where $\bar{\rho} \triangleq \rho+n_{\mathrm{r}}-m_{\mathrm{r}}$. Note that $\bar{\rho}$ is an upper bound on the relative degree of the cascaded system $G(s) \hat{G}_{\mathrm{r}}(s)$. Therefore, the parameter-dependent dynamic compensator is

$$
\hat{G}_{k}(s)=\frac{\delta k^{F_{\bar{\rho}+2}} \hat{z}_{\mathrm{r}}(s) \hat{z}(s)}{p_{\mathrm{r}}(s)\left[s^{\bar{\rho}}+k^{f_{\bar{\rho}, \bar{\rho}}} b_{\bar{\rho}} s^{\bar{\rho}-1}+k^{f_{\bar{\rho}, \bar{\rho}-1}} b_{\bar{\rho}-1} s^{\bar{\rho}-2}+\cdots+k^{f_{\bar{\rho}, 2}} b_{2} s+k^{f_{\bar{\rho}, 1}} b_{1}\right]}
$$

where $k, b_{1}, \ldots, b_{\bar{\rho}}$ are real, and $\hat{z}(s)$ is a degree $\bar{\rho}-1$ monic polynomial. Then closed-loop system (1), (2), (4), and (13) is

$$
y_{e}=\left[\begin{array}{ll}
\tilde{G}_{k, 1}(s) & \tilde{G}_{k, 2}(s)
\end{array}\right]\left[\begin{array}{l}
y_{\mathrm{r}} \\
w
\end{array}\right]
$$

where

$$
\begin{gathered}
\tilde{G}_{k, 1}(s) \triangleq \frac{1}{1+G(s) \hat{G}_{k}(s)}=\frac{\tilde{z}_{k, 1}(s)}{\tilde{p}_{k}(s)} \\
\tilde{G}_{k, 2}(s) \triangleq \frac{-G(s)}{1+G(s) \hat{G}_{k}(s)}=\frac{\tilde{z}_{k, 2}(s)}{\tilde{p}_{k}(s)}
\end{gathered}
$$


and

$$
\begin{gathered}
\tilde{z}_{k, 1}(s) \triangleq p_{\mathrm{r}}(s) p(s)\left[s^{\bar{\rho}}+k^{f_{\bar{\rho}, \bar{\rho}}} b_{\bar{\rho}} s^{\bar{\rho}-1}+k^{f_{\bar{\rho}, \bar{\rho}-1}} b_{\bar{\rho}-1} s^{\bar{\rho}-2}+\cdots+k^{f_{\bar{\rho}, 2}} b_{2} s+k^{f_{\bar{\rho}, 1}} b_{1}\right] \\
\tilde{z}_{k, 2}(s) \triangleq-\delta \beta p_{\mathrm{r}}(s) z(s)\left[s^{\bar{\rho}}+k^{f_{\bar{\rho}, \bar{\rho}}} b_{\bar{\rho}} s^{\bar{\rho}-1}+k^{f_{\bar{\rho}, \bar{\rho}-1}} b_{\bar{\rho}-1} s^{\bar{\rho}-2}+\cdots+k^{f_{\bar{\rho}, 2}} b_{2} s+k^{f_{\bar{\rho}, 1}} b_{1}\right] \\
\tilde{p}_{k}(s) \triangleq \\
p_{\mathrm{r}}(s) p(s) s^{\bar{\rho}}+k^{f_{\bar{\rho}, \bar{\rho}}} b_{\bar{\rho}} p_{\mathrm{r}}(s) p(s) s^{\bar{\rho}-1}+k^{f_{\bar{\rho}, \bar{\rho}-1}} b_{\bar{\rho}-1} p_{\mathrm{r}}(s) p(s) s^{\bar{\rho}-2} \\
+\cdots+k^{f_{\bar{\rho}, 1}} b_{1} p_{\mathrm{r}}(s) p(s)+k^{F_{\bar{\rho}+2}} \beta z(s) \hat{z}_{\mathrm{r}}(s) \hat{z}(s)
\end{gathered}
$$

\section{Theorem 4.1}

Consider the closed-loop system (14)-(19). Assume that the dynamic compensators $\hat{G}_{\mathrm{r}}(s)$ and $\hat{G}_{k, \bar{\rho}}(s)$ are minimum phase, that is, assume that the polynomials $\hat{z}_{\mathrm{r}}(s)$ and $\hat{z}(s)$ are Hurwitz. Furthermore, assume that the polynomials

$$
B_{\bar{\rho}-2}(s) \triangleq s^{3}+b_{\bar{\rho}} s^{2}+b_{\bar{\rho}-1} s+b_{0}
$$

and, for $i=0,1, \ldots, \bar{\rho}-3$,

$$
B_{i}(s) \triangleq b_{i+3} s^{3}+b_{i+2} s^{2}+b_{i+1} s+b_{0}
$$

are Hurwitz. Then the following statements hold:

(i) $\tilde{p}_{k}(s)$ is high-gain Hurwitz, and thus $\tilde{G}_{k, 1}(s)$ and $\tilde{G}_{k, 2}(s)$ are high-gain stable.

(ii) As $k \rightarrow \infty, m+m_{\mathrm{r}}+\bar{\rho}-1$ roots of $\tilde{p}_{k}(s)$ converge to the roots of $z(s) \hat{z}_{\mathrm{r}}(s) \hat{z}(s)$ and the real parts of the remaining $r+n_{\mathrm{r}}-m_{\mathrm{r}}+1$ roots approach $-\infty$.

(iii) There exists $k_{\mathrm{s}}>0$ such that, for all $k \geqslant k_{\mathrm{s}}, \lim _{t \rightarrow \infty} y_{e}(t)=0$.

\section{Proof}

Statements (i) and (ii) follow from applying Theorem 3.1 to the cascade $G(s) \hat{G}_{\mathrm{r}}(s)$. Specifically, define $\bar{G}(s) \triangleq G(s) \hat{G}_{\mathrm{r}}(s)$. Since $\hat{z}_{\mathrm{r}}(s)$ is Hurwitz, it follows that $\bar{G}(s)$ satisfies assumptions (I)-(VI) where $\bar{\rho}$ is an upper bound on the relative degree of $\bar{G}(s)$. Furthermore, $\tilde{p}_{k}(s)$ is the closed-loop parameter-dependent characteristic polynomial of $\bar{G}(s)$ connected in feedback with the controller $\hat{G}_{k, \bar{\rho}}(s)$. Then according to Theorem 3.1, $\tilde{p}_{k}(s)$ is high-gain Hurwitz, and, as $k \rightarrow$ $\infty, m+m_{\mathrm{r}}+\bar{\rho}-1$ roots of $\tilde{p}_{k}(s)$ converge to the roots of $z(s) \hat{z}_{\mathrm{r}}(s) \hat{z}(s)$ and the real parts of the remaining $r+n_{\mathrm{r}}-m_{\mathrm{r}}+1$ roots approach $-\infty$.

Now, we show part (iii). Letting $\mathscr{L}(\cdot)$ denote the Laplace operator, the final value theorem implies

$$
\begin{aligned}
& \lim _{t \rightarrow \infty} y_{e}(t)=\lim _{s \rightarrow 0} s \mathscr{L}\left(y_{e}(t)\right) \\
& =\lim _{s \rightarrow 0} s\left[\tilde{G}_{k, 1}(s) \tilde{G}_{k, 2}(s)\right]\left[\begin{array}{c}
\mathscr{L}\left(y_{\mathrm{r}}(t)\right) \\
\mathscr{L}(w(t))
\end{array}\right] \\
& =\lim _{s \rightarrow 0} s\left[\begin{array}{ll}
\frac{\tilde{z}_{k, 1}(s)}{\tilde{p}_{k}(s)} & \frac{\tilde{z}_{k, 2}(s)}{\tilde{p}_{k}(s)}
\end{array}\right]\left[\begin{array}{c}
\frac{z_{\mathrm{r}}(s)}{p_{\mathrm{r}}(s)} \\
\frac{z_{w}(s)}{p_{\mathrm{r}}(s)}
\end{array}\right]
\end{aligned}
$$




$$
\begin{aligned}
= & \lim _{s \rightarrow 0} s\left[\frac{p_{\mathrm{r}}(s) p(s)\left[s^{\bar{\rho}}+k^{f_{\bar{\rho}, \bar{\rho}}} b_{\bar{\rho}} s^{\bar{\rho}-1}+\cdots+k^{f_{\bar{\rho}, 1}} b_{1}\right]}{\tilde{p}_{k}(s)} \frac{z_{\mathrm{r}}(s)}{p_{\mathrm{r}}(s)}\right. \\
& \left.+\frac{-\delta \beta p_{\mathrm{r}}(s) z(s)\left[s^{\bar{\rho}}+k^{f_{\bar{p}, \bar{\rho}}} b_{\bar{\rho}} s^{\bar{\rho}-1}+\cdots+k^{f_{\bar{p}, 1}} b_{1}\right]}{\tilde{p}_{k}(s)} \frac{z_{w}(s)}{p_{\mathrm{r}}(s)}\right] \\
= & \lim _{s \rightarrow 0} s \frac{\left[p(s) z_{\mathrm{r}}(s)-\delta \beta z(s) z_{w}(s)\right]\left[s^{\bar{\rho}}+k^{f_{\bar{p}, \bar{\rho}}} b_{\bar{\rho}} s^{\bar{\rho}-1}+\cdots+k^{f_{\bar{\rho}, 1}} b_{1}\right]}{\tilde{p}_{k}(s)}
\end{aligned}
$$

where $\mathscr{L}\left(y_{\mathrm{r}}(t)\right)=z_{\mathrm{r}}(s) / p_{\mathrm{r}}(s), \mathscr{L}(w(t))=z_{w}(s) / p_{\mathrm{r}}(s)$, and $z_{\mathrm{r}}(s)$ and $z_{w}(s)$ are polynomials. Since $\tilde{p}_{k}(s)$ is high-gain Hurwitz, there exists $k_{\mathrm{s}}>0$ such that, for all $k \geqslant k_{\mathrm{s}}, \tilde{p}_{k}(s)$ is Hurwitz. Then (22) implies, for all $k \geqslant k_{\mathrm{s}}, \lim _{t \rightarrow \infty} y_{e}(t)=0$.

\section{PARAMETER-MONOTONIC ADAPTIVE COMMAND FOLLOWING AND DISTURBANCE REJECTION FOR SYSTEMS WITH UNKNOWN-BUT- BOUNDED RELATIVE DEGREE}

Although Theorem 4.1 guarantees the existence of a strictly proper parameter-dependent dynamic compensator (13) for asymptotic command following and disturbance rejection, the stabilizing threshold $k_{\mathrm{s}}$ is unknown. In this section, we introduce a parameter-monotonic adaptive law for the parameter $k$ and present our main result. To complete our analysis, we construct state space realizations for the open-loop system (1) and (2) and the compensator (4) and (13). Let the system (1) and(2) have the minimal state space realization

$$
\dot{x}=A x+B(u+w), \quad y=C x
$$

where $A \in \mathbb{R}^{n \times n}, B \in \mathbb{R}^{n \times 1}$, and $C \in \mathbb{R}^{1 \times n}$.

Next, consider the controller $\hat{G}_{k}(s)=\hat{G}_{\mathrm{r}}(s) \hat{G}_{k, \bar{\rho}}(s)$ given by (4) and (13) and write

$$
\hat{z}(s)=s^{\bar{\rho}-1}+\hat{z}_{\bar{\rho}-2} s^{\bar{\rho}-2}+\cdots+\hat{z}_{1} s+\hat{z}_{0}
$$

so that $\hat{G}_{k}(s)$ has the state space realization

$$
\dot{\hat{x}}=\hat{A}(k) \hat{x}+\hat{B} y_{e}, \quad u=\hat{C}(k) \hat{x}
$$

where $\hat{A}(k) \in \mathbb{R}^{\left(n_{\mathrm{r}}+\bar{\rho}\right) \times\left(n_{\mathrm{r}}+\bar{\rho}\right)}, \hat{B} \in \mathbb{R}^{\left(n_{\mathrm{r}}+\bar{\rho}\right) \times 1}$, and $\hat{C} \in \mathbb{R}^{1 \times\left(n_{\mathrm{r}}+\bar{\rho}\right)}$ are given by

$$
\begin{gathered}
\hat{A}(k) \triangleq\left[\begin{array}{cc}
\hat{A}_{\mathrm{r}} & \hat{B}_{\mathrm{r}} \hat{C}_{\bar{\rho}}(k) \\
0 & \hat{B}_{\bar{\rho}}(k)
\end{array}\right], \quad \hat{B} \triangleq\left[\begin{array}{c}
0 \\
\hat{A}_{\bar{\rho}}
\end{array}\right] \\
\hat{C}(k) \triangleq\left[\hat{C}_{\mathrm{r}} \quad \hat{D}_{\mathrm{r}} \hat{C}_{\bar{\rho}}(k)\right]
\end{gathered}
$$

where

$$
\hat{A}_{\bar{\rho}}(k) \triangleq\left[\begin{array}{cccc}
-k^{f_{\bar{\rho}, \bar{\rho}}} b_{\bar{\rho}} & 1 & \cdots & 0 \\
\vdots & & \ddots & \vdots \\
-k^{f_{\bar{\rho}, 2}} b_{2} & 0 & & 1 \\
-k^{f_{\bar{\rho}, 1}} b_{1} & 0 & \cdots & 0
\end{array}\right], \quad \hat{B}_{\bar{\rho}} \triangleq\left[\begin{array}{c}
1 \\
\hat{z}_{\bar{\rho}-2} \\
\vdots \\
\hat{z}_{0}
\end{array}\right]
$$




$$
\hat{C}_{\bar{\rho}}(k) \triangleq\left[\begin{array}{llll}
\delta k^{F_{\bar{\rho}+2}} & 0 & \cdots & 0
\end{array}\right]
$$

is a realization of $\hat{G}_{k, \bar{\rho}}(s)$ and $\left(\hat{A}_{\mathrm{r}}, \hat{B}_{\mathrm{r}}, \hat{C}_{\mathrm{r}}, \hat{D}_{\mathrm{r}}\right)$ is a minimal realization of $\hat{G}_{\mathrm{r}}(s)$. Note that, for all non-zero $k \in \mathbb{R},\left(\hat{A}_{\bar{\rho}}(k), \hat{C}_{\bar{\rho}}(k)\right)$ is observable. The closed-loop system (23) and (25)-(29) is

$$
\begin{gathered}
\dot{\tilde{x}}=\tilde{A}(k) \tilde{x}+\tilde{B} u_{\mathrm{r}} \\
y_{e}=\tilde{C} \tilde{x}+\tilde{D} u_{\mathrm{r}}
\end{gathered}
$$

where

$$
\begin{gathered}
\tilde{A}(k) \triangleq\left[\begin{array}{cc}
A & B \hat{C}(k) \\
-\hat{B} C & \hat{A}(k)
\end{array}\right], \quad \tilde{B} \triangleq\left[\begin{array}{ll}
0 & B \\
\hat{B} & 0
\end{array}\right], \quad \tilde{x} \triangleq\left[\begin{array}{l}
x \\
\hat{x}
\end{array}\right] \\
\tilde{C} \triangleq\left[\begin{array}{ll}
-C & 0
\end{array}\right], \quad \tilde{D} \triangleq\left[\begin{array}{ll}
1 & 0
\end{array}\right]
\end{gathered}
$$

Now we present the main result of this paper, namely direct adaptive command following and disturbance rejection for minimum-phase systems with unknown-but-bounded relative degree.

\section{Theorem 5.1}

Consider the closed-loop system (30)-(33) consisting of the open-loop system (23) with unknown relative degree $r$ satisfying $1 \leqslant r \leqslant \rho$, and the feedback controller (25)-(29). Furthermore, consider the parameter-monotonic adaptive law

$$
\dot{k}(t)=\gamma \mathrm{e}^{-\alpha k(t)} y_{e}^{2}(t)
$$

where $\gamma>0$ and $\alpha>0$. Assume that the dynamic compensators $\hat{G}_{\mathrm{r}}(s)$ and $\hat{G}_{k, \bar{\rho}}(s)$ are minimum phase, that is, assume that the polynomials $\hat{z}_{\mathrm{r}}(s)$ and $\hat{z}(s)$ are Hurwitz. Furthermore, assume that the polynomials $B_{0}(s), \ldots, B_{\bar{\rho}-2}(s)$ given by $(20)$ and $(21)$ are Hurwitz. Then, for all initial conditions $\tilde{x}(0)$ and $k(0)>0, k(t)$ converges and $\lim _{t \rightarrow \infty} y_{e}(t)=0$.

\section{Proof}

The closed-loop system (30)-(33) with the inputs $y_{\mathrm{r}}$ and $w$ generated by the linear system (3) can be written as

$$
\begin{gathered}
\dot{x}_{c}(t)=A_{c}(k(t)) x_{c}(t) \\
y_{e}(t)=C_{c} x_{c}(t)
\end{gathered}
$$

where

$$
A_{c}(k) \triangleq\left[\begin{array}{cc}
\tilde{A}(k) & \tilde{B} C_{\mathrm{r}} \\
0 & A_{\mathrm{r}}
\end{array}\right], \quad C_{c} \triangleq\left[\begin{array}{ll}
\tilde{C} & \tilde{D} C_{\mathrm{r}}
\end{array}\right], \quad x_{c}(t) \triangleq\left[\begin{array}{c}
\tilde{x}(t) \\
x_{\mathrm{r}}(t)
\end{array}\right]
$$

Since (34)-(36) is locally Lipschitz, it follows that the solution to (34)-(36) exists and is unique locally, that is, there exists $t_{e}>0$ such that $\left(x_{c}(t), k(t)\right)$ exists on the interval $\left[0, t_{e}\right)$.

First, we show that if $x_{c}(t)$ escapes at $t_{e}$, then $k(t)$ escapes at $t_{e}$. Assume that $k(t)$ does not escape at $t_{e}$. Let $k\left(t_{e}\right) \triangleq \lim _{t \rightarrow t_{e}} k(t)$ so that $k(t)$ is continuous on $\left[0, t_{e}\right]$. Then $\dot{q}(t)=A_{c}(k(t)) q(t)$ is 
a linear time-varying differential equation on $\left[0, t_{e}\right]$, where $A_{c}(k(t))$ is continuous in $t$. The solution to $\dot{q}(t)=A_{c}(k(t)) q(t)$ exists and is unique on [0, $\left.t_{e}\right]$ [19]. Therefore, $q(t)$ does not escape at $t_{e}$ and, by uniqueness, $x_{c}(t)=q(t)$ on $\left[0, t_{e}\right]$. Consequently, $x_{c}(t)$ does not escape at $t_{e}$. Hence, if $x_{c}(t)$ escapes at $t_{e}$, then $k(t)$ escapes at $t_{e}$.

Now, we show that $k(t)$ converges. For contradiction, suppose that $k(t)$ diverges to infinity at $t_{e}$. Theorem 4.1 implies that there exists $k_{\mathrm{s}}>0$, such that for all $k \geqslant k_{\mathrm{s}}, \tilde{A}(k)$ is asymptotically stable and $\lim _{t \rightarrow \infty} y_{e}(t)=0$. Since, for all $k \geqslant k_{\mathrm{s}}, \tilde{A}(k)$ is asymptotically stable and $\lim _{t \rightarrow \infty} y_{e}(t)=0$, it follows from Lemma A.2 that there exists $P:\left[k_{\mathrm{s}}, \infty\right) \rightarrow \mathbb{R}^{\left(n+2 n_{\mathrm{r}}+\bar{\rho}\right) \times\left(n+2 n_{\mathrm{r}}+\bar{\rho}\right)}$ and $Q:\left[k_{\mathrm{s}}, \infty\right) \rightarrow \mathbb{R}^{\left(n+2 n_{\mathrm{r}}+\bar{\rho}\right) \times\left(n+2 n_{\mathrm{r}}+\bar{\rho}\right)}$ such that the entries of $P$ and $Q$ are real rational functions and, for all $k \geqslant k_{\mathrm{s}}, P(k)$ is positive definite, $Q(k)$ is positive semidefinite, and

$$
A_{c}^{\mathrm{T}}(k) P(k)+P(k) A_{c}(k)=-Q(k)-\gamma C_{c}^{\mathrm{T}} C_{c}
$$

Lemma A.3 implies that there exists $k_{2} \geqslant k_{\mathrm{s}}$ such that, for all $k \geqslant k_{2}, \alpha P(k)>\partial P(k) / \partial k$. Since $k(t)$ diverges to infinity at $t_{e}$, there exists $t_{2}<t_{e}$ such that $k\left(t_{2}\right)=k_{2}$.

For all $t \in\left[t_{2}, t_{e}\right)$, define

$$
V_{0}\left(x_{c}, k\right) \triangleq \mathrm{e}^{-\alpha k(t)} x_{c}^{\mathrm{T}} P(k) x_{c}
$$

Note that $V_{0}\left(x_{c}, k\right)$ is not to be considered a candidate Lyapunov function but is non-negative for all $k \geqslant k_{\mathrm{s}}$ and for all $x \in \mathbb{R}^{n+2 n_{\mathrm{r}}+\bar{\rho}}$. For all $t \in\left[t_{2}, t_{e}\right)$ the derivative of $V_{0}\left(x_{c}, k\right)$ along trajectories of (34)-(36) satisfies

$$
\begin{aligned}
\dot{V}_{0}\left(x_{c}, k\right) & =-\mathrm{e}^{-\alpha k(t)} x_{c}^{\mathrm{T}}\left[A_{c}^{\mathrm{T}}(k) P(k)+P(k) A_{c}(k)\right] x_{c}-\alpha \dot{k} \mathrm{e}^{-\alpha k} x_{c}^{\mathrm{T}} P(k) x_{c}+\dot{k} \mathrm{e}^{-\alpha k} x_{c}^{\mathrm{T}} \frac{\partial P(k)}{\partial k} x_{c} \\
& =-\mathrm{e}^{-\alpha k} x_{c}^{\mathrm{T}} Q(k) x_{c}-\gamma \mathrm{e}^{-\alpha k} x_{c}^{\mathrm{T}} C_{c}^{\mathrm{T}} C_{c} x_{c}-\dot{k} \mathrm{e}^{-\alpha k} x_{c}^{\mathrm{T}}\left[\alpha P(k)-\frac{\partial P(k)}{\partial k}\right] x_{c} \\
& \leqslant-\mathrm{e}^{-\alpha k} x_{c}^{\mathrm{T}} Q(k) x_{c}-\gamma \mathrm{e}^{-\alpha k} y_{e}^{2} \\
& \leqslant-\gamma \mathrm{e}^{-\alpha k} y_{e}^{2}=-\dot{k}
\end{aligned}
$$

Integrating (37) from $t_{2}$ to $t<t_{e}$ and solving for $k(t)$ yields

$$
k(t) \leqslant V_{0}\left(x_{c}\left(t_{2}\right), k_{2}\right)+k_{2}-\mathrm{e}^{-\alpha k(t)} x_{c}^{\mathrm{T}}(t) P(k(t)) x_{c}(t) \leqslant V_{0}\left(x_{c}\left(t_{2}\right), k_{2}\right)+k_{2}
$$

for $t \in\left[t_{2}, t_{e}\right)$. Hence, $k(\cdot)$ is bounded on $\left[0, t_{e}\right)$, which is a contradiction. Therefore, the solution to (34)-(36) exists and is unique on all finite intervals. Then integrating (37) from $t_{2}$ to $t<\infty$ yields (38) for $t \in\left[t_{2}, \infty\right)$. Therefore, $k(\cdot)$ is bounded on $[0, \infty)$. Since $k(t)$ is non-decreasing, $k_{\infty} \triangleq \lim _{t \rightarrow \infty} k(t)$ exists.

Since for all $t>0, k(t)<k_{\infty}$, it follows that

$$
\gamma \mathrm{e}^{-\alpha k_{\infty}} \int_{0}^{t} y_{e}^{2}(\tau) \mathrm{d} \tau \leqslant \gamma \int_{0}^{t} \mathrm{e}^{-\alpha k(\tau)} y_{e}^{2}(\tau) \mathrm{d} \tau=k(t)-k(0)<k_{\infty}-k(0)
$$

and thus $y_{e}(\cdot)$ is square integrable on $[0, \infty)$. This property will be used later. 
Next, we show that, for all $k>0$, the pair $(\tilde{A}(k), \tilde{C})$ is detectable. Let $\lambda$ be an element of the closed right half plane. Then

$$
\begin{aligned}
\operatorname{rank}\left[\begin{array}{c}
\tilde{A}(k)-\lambda I \\
\tilde{C}
\end{array}\right] & =\operatorname{rank}\left[\begin{array}{cc}
A-\lambda I & B \hat{C}(k) \\
-\hat{B} C & \hat{A}(k)-\lambda I \\
-C & 0
\end{array}\right] \\
& =\operatorname{rank}\left[\begin{array}{cc}
A-\lambda I & B \hat{C}(k) \\
C & 0 \\
0 & \hat{A}(k)-\lambda I
\end{array}\right] \\
& =\operatorname{rank}\left[\begin{array}{ccc}
A-\lambda I & B & 0 \\
C & 0 & 0 \\
0 & 0 & I_{n_{\mathrm{r}}+\bar{\rho}}
\end{array}\right]\left[\begin{array}{cc}
I_{n} & 0 \\
0 & \hat{C}(k) \\
0 & \hat{A}(k)-\lambda I
\end{array}\right]
\end{aligned}
$$

Since $(A, B, C)$ is a minimal realization of the minimum-phase plant (1) and (2), it follows that

$$
\left[\begin{array}{ccc}
A-\lambda I & B & 0 \\
C & 0 & 0 \\
0 & 0 & I_{n_{\mathrm{r}}+\bar{\rho}}
\end{array}\right]
$$

is non-singular. Thus,

$$
\begin{aligned}
\operatorname{rank}\left[\begin{array}{c}
\tilde{A}(k)-\lambda I \\
\tilde{C}
\end{array}\right] & =\operatorname{rank}\left[\begin{array}{cc}
I_{n} & 0 \\
0 & \hat{C}(k) \\
0 & \hat{A}(k)-\lambda I
\end{array}\right] \\
& =\operatorname{rank}\left[\begin{array}{ccc}
I_{n} & 0 & 0 \\
0 & \hat{C}_{\mathrm{r}} & \hat{D}_{\mathrm{r}} \hat{C}_{\bar{\rho}}(k) \\
0 & \hat{A}_{\mathrm{r}}-\lambda I & \hat{B}_{\mathrm{r}} \hat{C}_{\bar{\rho}}(k) \\
0 & 0 & \hat{A}_{\bar{\rho}}(k)-\lambda I
\end{array}\right]
\end{aligned}
$$

which implies

$$
\operatorname{rank}\left[\begin{array}{c}
\tilde{A}(k)-\lambda I \\
\tilde{C}
\end{array}\right]=\operatorname{rank}\left[\begin{array}{ccc}
I_{n} & 0 & 0 \\
0 & \hat{A}_{\mathrm{r}}-\lambda I & \hat{B}_{\mathrm{r}} \hat{C}_{\bar{\rho}}(k) \\
0 & \hat{C}_{\mathrm{r}} & \hat{D}_{\mathrm{r}} \hat{C}_{\bar{\rho}}(k) \\
0 & 0 & \hat{A}_{\bar{\rho}}(k)-\lambda I
\end{array}\right]
$$




$$
=\operatorname{rank}\left[\begin{array}{cccc}
I_{n} & 0 & 0 & 0 \\
0 & \hat{A}_{\mathrm{r}}-\lambda I & \hat{B}_{\mathrm{r}} & 0 \\
0 & \hat{C}_{\mathrm{r}} & \hat{D}_{\mathrm{r}} & 0 \\
0 & 0 & 0 & I_{\bar{\rho}}
\end{array}\right]\left[\begin{array}{ccc}
I_{n} & 0 & 0 \\
0 & I_{n_{\mathrm{r}}} & 0 \\
0 & 0 & \hat{C}_{\bar{\rho}}(k) \\
0 & 0 & \hat{A}_{\bar{\rho}}(k)-\lambda I
\end{array}\right]
$$

Since $\left(\hat{A}_{\mathrm{r}}, \hat{B}_{\mathrm{r}}, \hat{C}_{\mathrm{r}}, \hat{D}_{\mathrm{r}}\right)$ is a minimal realization of the minimum-phase compensator $\hat{G}_{\mathrm{r}}(s)$, it follows that

$$
\left[\begin{array}{cccc}
I_{n} & 0 & 0 & 0 \\
0 & \hat{A}_{\mathrm{r}}-\lambda I & \hat{B}_{\mathrm{r}} & 0 \\
0 & \hat{C}_{\mathrm{r}} & \hat{D}_{\mathrm{r}} & 0 \\
0 & 0 & 0 & I_{\bar{\rho}}
\end{array}\right]
$$

is non-singular. Thus,

$$
\operatorname{rank}\left[\begin{array}{c}
\tilde{A}(k)-\lambda I \\
\tilde{C}
\end{array}\right]=\operatorname{rank}\left[\begin{array}{ccc}
I_{n} & 0 & 0 \\
0 & I_{n_{\mathrm{r}}} & 0 \\
0 & 0 & \hat{C}_{\bar{\rho}}(k) \\
0 & 0 & \hat{A}_{\bar{\rho}}(k)-\lambda I
\end{array}\right]
$$

Since, for all $k>0,\left(\hat{A}_{\bar{\rho}}(k), \hat{C}_{\bar{\rho}}(k)\right)$ is observable, it follows that, for all $k>0$

$$
\operatorname{rank}\left[\begin{array}{c}
\tilde{A}(k)-\lambda I \\
\tilde{C}
\end{array}\right]=n+n_{\mathrm{r}}+\bar{\rho}
$$

Therefore, for all $k>0,(\tilde{A}(k), \tilde{C})$ is detectable.

Next, we show that $\lim _{t \rightarrow \infty} y_{e}(t)=0$. Define $A_{\infty} \triangleq \tilde{A}\left(k_{\infty}\right)$. Since $\left(A_{\infty}, \tilde{C}\right)$ is detectable, it follows that there exists $L \in \mathbb{R}^{\left(n+n_{\mathrm{r}}+\bar{\rho}\right) \times 1}$ such that $A_{\mathrm{S}} \triangleq A_{\infty}+L \tilde{C}$ is asymptotically stable. Then adding and subtracting $A_{\mathrm{s}} x$ and $L \tilde{D} u_{\mathrm{r}}$ from (30) implies

$$
\dot{\tilde{x}}(t)=A_{\mathrm{s}} \tilde{x}(t)+\left[\tilde{A}(k(t))-A_{\infty}\right] \tilde{x}(t)+[\tilde{B}+L \tilde{D}] u_{\mathrm{r}}(t)-L\left[\tilde{C} \tilde{x}(t)+\tilde{D} u_{\mathrm{r}}(t)\right]
$$

which is equivalent to

$$
\dot{\tilde{x}}(t)=A_{\mathrm{s}} \tilde{x}(t)+\Delta(t) \tilde{x}(t)+J u_{\mathrm{r}}(t)-L y_{e}(t)
$$

where

$$
\begin{gathered}
\Delta(t) \triangleq \tilde{A}(k(t))-A_{\infty} \\
J \triangleq \tilde{B}+L \tilde{D}
\end{gathered}
$$

Since $A_{\mathrm{S}}$ is asymptotically stable, $\Delta(\cdot)$ is continuous, $\lim _{t \rightarrow \infty} \Delta(t)=0, u_{\mathrm{r}}(\cdot)$ is bounded on $[0, \infty)$, and $y_{e}(\cdot)$ is square integrable on $[0, \infty)$, it follows from Lemma B.1 that $\tilde{x}(\cdot)$ is bounded on $[0, \infty)$. 


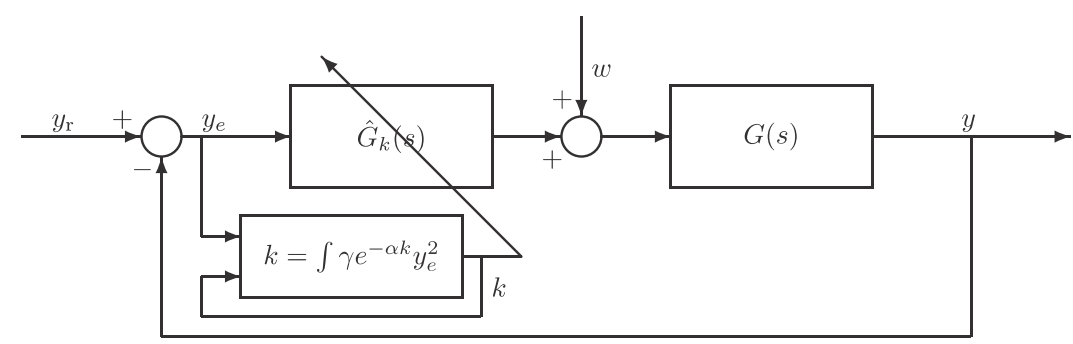

Figure 2. Adaptive controller for the command following and disturbance rejection problem.

Next, since $\tilde{A}(\cdot)$ is bounded, $\tilde{x}(\cdot)$ is bounded, and $u_{\mathrm{r}}(\cdot)$ is bounded, it follows from (30) that $\dot{\tilde{x}}(\cdot)$ is bounded. Since $\tilde{x}(\cdot), \dot{\tilde{x}}(\cdot), u_{\mathrm{r}}(\cdot)$, and $\dot{u}_{\mathrm{r}}(\cdot)$ are bounded, it follows from (30) and (31) that $y_{e}(\cdot)$ and $\dot{y}_{e}(\cdot)$ are bounded. Therefore, $\mathrm{d}\left(y_{e}^{2}(t)\right) / \mathrm{d} t=2 \dot{y}_{e}(t) y_{e}(t)$ is bounded, and thus $y_{e}^{2}(t)$ is uniformly continuous. Since $y_{e}^{2}(t)$ is uniformly continuous and $\lim _{t \rightarrow \infty} \int_{0}^{t} y_{e}^{2}(\tau) \mathrm{d} \tau$ exists, Barbalat's lemma implies that $\lim _{t \rightarrow \infty} y_{e}(t)=0$.

Figure 2 illustrates the adaptive controller presented in Theorem 5.1.

\section{SERIALLY CONNECTED MASS-SPRING-DAMPER}

Consider the three-mass serially connected mass-spring-damper system shown in Figure 3. The dynamics of the system are given by

$$
M \ddot{q}+C \dot{q}+K q=b(u+w)
$$

where

$$
\begin{gathered}
M \triangleq\left[\begin{array}{ccc}
m_{1} & & \\
& m_{2} & \\
& & m_{3}
\end{array}\right], \quad b \triangleq\left[\begin{array}{l}
1 \\
0 \\
0
\end{array}\right] \\
C \triangleq\left[\begin{array}{ccc}
c_{1}+c_{2} & -c_{2} & 0 \\
-c_{2} & c_{2}+c_{3} & -c_{3} \\
0 & -c_{3} & c_{3}+c_{4}
\end{array}\right] \\
K \triangleq\left[\begin{array}{ccc}
k_{1}+k_{2} & -k_{2} & 0 \\
-k_{2} & k_{2}+k_{3} & -k_{3} \\
0 & -k_{3} & k_{3}+k_{4}
\end{array}\right] \\
q \triangleq\left[\begin{array}{lll}
q_{1} & q_{2} & q_{3}
\end{array}\right]^{\mathrm{T}}
\end{gathered}
$$




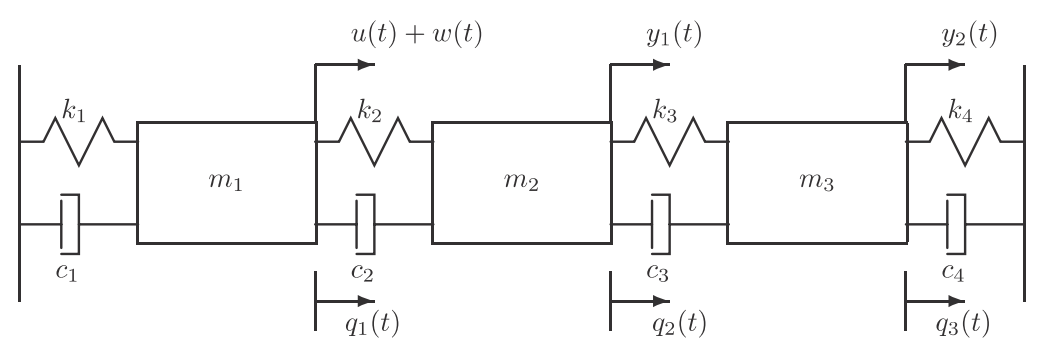

Figure 3. Three-mass serially connected mass-spring-damper system.

The masses are $m_{1}=1 \mathrm{~kg}, m_{2}=0.5 \mathrm{~kg}$, and $m_{3}=1 \mathrm{~kg}$; the damping coefficients are $c_{1}=c_{2}=$ $c_{3}=c_{4}=2 \mathrm{~kg} / \mathrm{s}$; and the spring constants are $k_{1}=2 \mathrm{~kg} / \mathrm{s}^{2}, k_{2}=4 \mathrm{~kg} / \mathrm{s}^{2}, k_{3}=1 \mathrm{~kg} / \mathrm{s}^{2}$, and $k_{4}=3 \mathrm{~kg} / \mathrm{s}^{2}$.

Our objective is to design an adaptive controller so that every SISO force-to-position transfer function of the system (39)-(43) can track a sinusoid of $\omega_{1}=11 \mathrm{rad} / \mathrm{s}$ and a step, while rejecting a sinusoid of $\omega_{2}=8 \mathrm{rad} / \mathrm{s}$ and a constant disturbance. Thus, the dynamics for tracking and disturbance rejection are given by the characteristic polynomial

$$
p_{\mathrm{r}}(s)=s\left(s^{2}+\omega_{1}^{2}\right)\left(s^{2}+\omega_{2}^{2}\right)
$$

All SISO force-to-position transfer functions of an asymptotically stable serially connected structure are known to be minimum phase [20]. Furthermore, [20] show that the relative degree of a SISO force-to-position transfer function for a serially connected structure is equal to the number of intervening masses plus two. For a three mass system, all force-to-position transfer functions have relative degree not exceeding four. Therefore, $\rho=4$ is an upper bound on the relative degree of the force-to-position transfer functions for a three-mass system. Lastly, it follows from [20] that all SISO force-to-position transfer functions of an asymptotically stable serially connected structure have positive high-frequency gain, so let $\delta=1$. Next, let us assume that the upper bound on the magnitude of the high-frequency gain is $b_{0}=10$. Then all SISO force-to-position transfer functions satisfy assumptions (I)-(VI).

Next, consider the parameter-dependent transfer function (13) where $\bar{\rho}=4$, which is given by

$$
\hat{G}_{k}(s)=\frac{k^{8} \hat{z}_{\mathrm{r}}(s) \hat{z}(s)}{p_{\mathrm{r}}(s)\left[s^{4}+k^{3} b_{4} s^{3}+k^{5} b_{3} s^{2}+k^{6} b_{2} s+k^{7} b_{1}\right]}
$$

To satisfy the assumptions of Theorem 4.1 the design parameters are chosen to be

$$
\begin{gathered}
\hat{z}_{\mathrm{r}}(s)=(s+2)(s+4)(s+6)(s+8)(s+10) \\
\hat{z}(s)=(s+15)(s+20)(s+25) \\
b_{4}=4, \quad b_{3}=4, \quad b_{2}=12, \quad b_{1}=4
\end{gathered}
$$

Then, the adaptive controller considered in Theorem 5.1 is given by the adaptive law

$$
\dot{k}(t)=\gamma \mathrm{e}^{-\alpha k(t)} y_{e}^{2}(t)
$$


and (25), where

$$
\begin{gathered}
\hat{A}_{\mathrm{r}}(k) \triangleq\left[\begin{array}{ccccc}
0 & 1 & 0 & 0 & 0 \\
0 & 0 & 1 & 0 & 0 \\
0 & 0 & 0 & 1 & 0 \\
0 & 0 & 0 & 0 & 1 \\
0 & -7744 & 0 & -185 & 0
\end{array}\right], \quad \hat{B}_{\mathrm{r}} \triangleq\left[\begin{array}{l}
0 \\
0 \\
0 \\
0 \\
1
\end{array}\right] \\
\hat{C}_{\mathrm{r}}(k) \triangleq\left[\begin{array}{lllll}
3840-3360 & 1800 & 155 & 30
\end{array}\right], \quad \hat{D}_{\mathrm{r}} \triangleq 1 \\
\hat{A}_{\bar{\rho}}(k) \triangleq\left[\begin{array}{cccc}
-4 k^{3} & 1 & 0 & 0 \\
-4 k^{5} & 0 & 1 & 0 \\
-12 k^{6} & 0 & 0 & 1 \\
-4 k^{7} & 0 & 0 & 0
\end{array}\right], \quad \hat{B}_{\bar{\rho}} \triangleq\left[\begin{array}{c}
1 \\
60 \\
1175 \\
7500
\end{array}\right] \\
\hat{C}_{\bar{\rho}}(k) \triangleq\left[\begin{array}{llll}
k^{8} & 0 & 0 & 0
\end{array}\right], \quad \gamma=1, \quad \alpha=0.1
\end{gathered}
$$

Now, we assume that the sensor is placed so that the position of $m_{2}$ is the output of the forceto-position system we are trying to control. This system is

$$
y_{1}=G_{1}(s)(u+w)
$$

where

$$
G_{1}(s) \triangleq \frac{4 s^{3}+24 s^{2}+48 s+32}{s^{6}+16 s^{5}+84 s^{4}+224 s^{3}+330 s^{2}+280 s+100}
$$

Furthermore, let us assume that the reference and disturbance signals are

$$
\begin{gathered}
y_{\mathrm{r}}(t)=10 \sin \left(\omega_{1} t\right)+5 \\
w(t)=7 \cos \left(\omega_{2} t\right)-8
\end{gathered}
$$

The mass-spring-damper system (49) and (50) is simulated with the initial conditions $q(0)=\left[\begin{array}{lll}-0.5 & 0.25 & 1.0\end{array}\right]^{\mathrm{T}} \mathrm{m}$ and $\dot{q}(0)=\left[\begin{array}{lll}0.1 & -0.2 & 0.3\end{array}\right]^{\mathrm{T}} \mathrm{m} / \mathrm{s}$. The adaptive controller $(25)$ and (44)-(48) is implemented in the feedback loop with $y_{e}(t)=y_{\mathrm{r}}(t)-y_{1}(t)$ and initial conditions $\hat{x}(0)=0$ and $k(0)=25$. Figure 4 shows that $y_{1}(t)$ asymptotically tracks $y_{\mathrm{r}}(t)$, that is, $y_{e}(t)$ converges to zero. Figure 5 shows that $u(t)$ is bounded and $k(t)$ converges to approximately 42.2 .

Now let us assume that the position sensor is placed on the third mass instead of the second mass. Then, we are trying to control the force-to-position system

$$
y_{2}=G_{2}(s)(u+w)
$$

where

$$
G_{2}(s) \triangleq \frac{8 s^{2}+20 s+8}{s^{6}+16 s^{5}+84 s^{4}+224 s^{3}+330 s^{2}+280 s+100}
$$



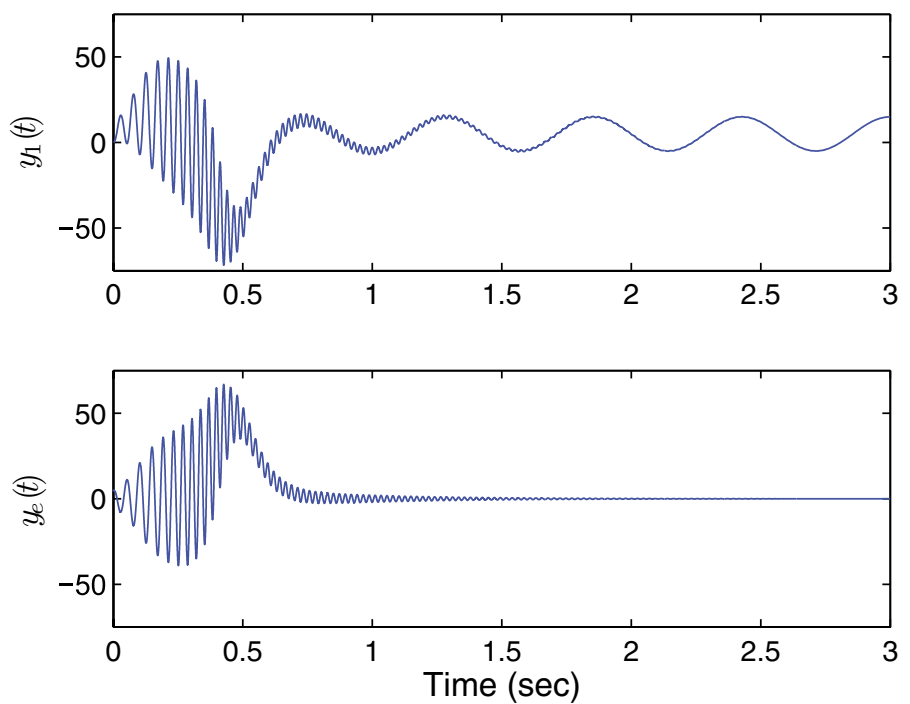

Figure 4. The output $y_{1}(t)$ asymptotically tracks the reference $y_{\mathrm{r}}(t)$, so $y_{e}(t)$ converges to zero.
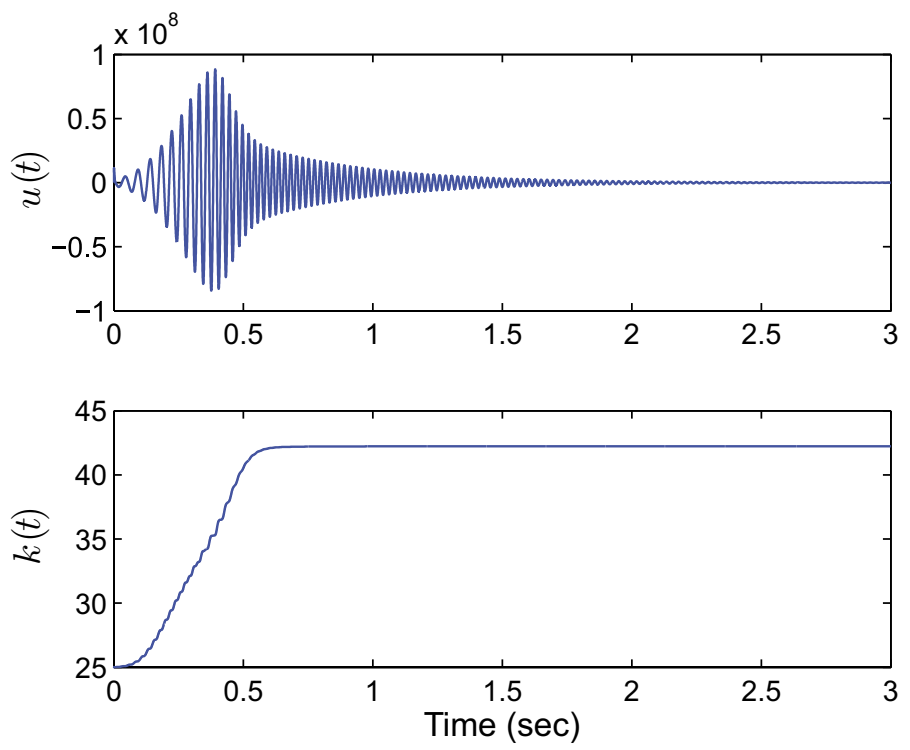

Figure 5. The control $u(t)$ is bounded and the gain $k(t)$ converges to approximately 42.2 .

Note that $G_{2}(s)$ has relative degree 4 instead of 3 . As before, the reference and disturbance signals are given by (51) and (52). The mass-spring-damper system (53) and (54) is simulated with the initial conditions $q(0)=\left[\begin{array}{lll}-0.5 & 0.25 & 1.0\end{array}\right]^{\mathrm{T}} \mathrm{m}$ and $\dot{q}(0)=\left[\begin{array}{lll}0.1 & -0.2 & 0.3\end{array}\right]^{\mathrm{T}} \mathrm{m} / \mathrm{s}$. The adaptive controller (25) and (44)-(48) is implemented in the feedback loop with 

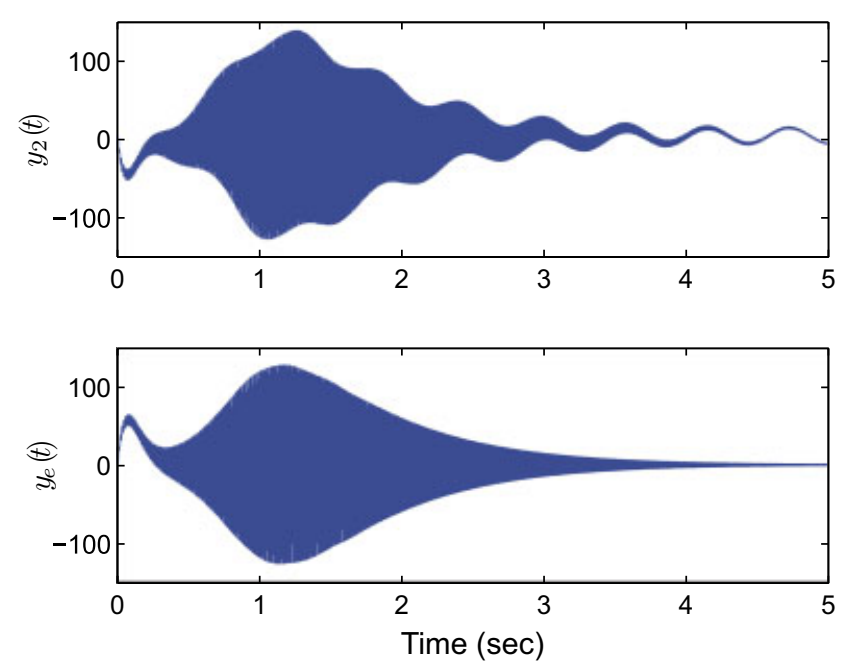

Figure 6. The output $y_{2}(t)$ asymptotically tracks the reference $y_{\mathrm{r}}(t)$, so $y_{e}(t)$ converges to zero.
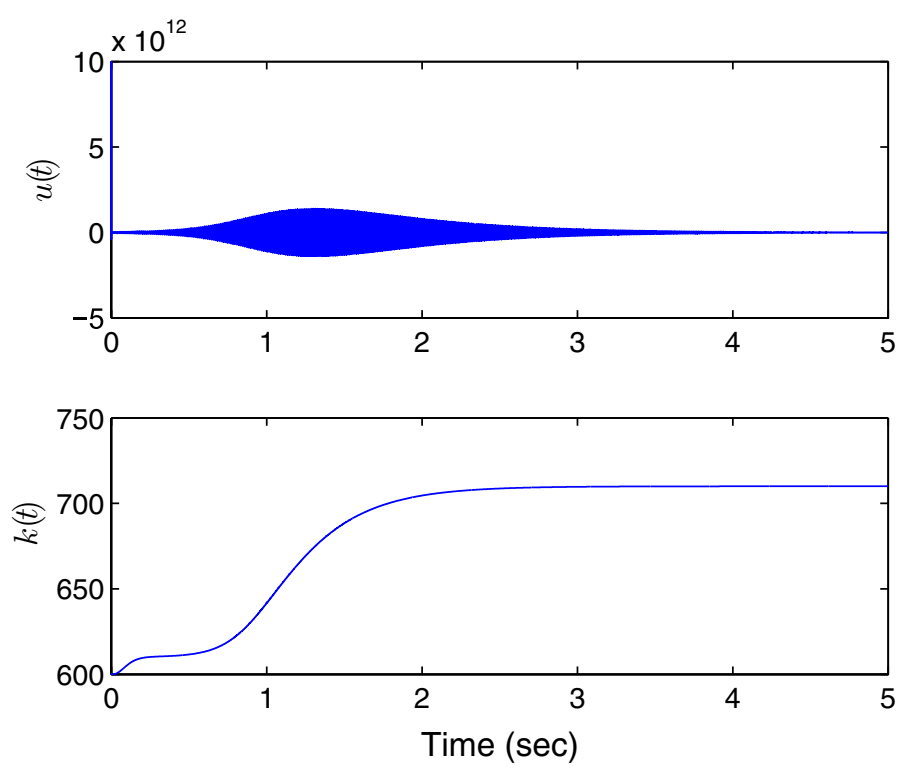

Figure 7. The control $u(t)$ is bounded and the gain $k(t)$ converges to approximately 711 .

$y_{e}(t)=y_{\mathrm{r}}(t)-y_{2}(t)$ and initial conditions $\hat{x}(0)=0$ and $k(0)=600$. Figures 6 and 7 show that $y_{e}(t)$ converges to zero and $k(t)$ converges to approximately 711 .

Lastly, to demonstrate the adaptive controller for an open-loop unstable system, assume that $c_{1}=-2 \mathrm{~kg} / \mathrm{s}$ instead of $2 \mathrm{~kg} / \mathrm{s}$. Then the damper $c_{1}$ supplies energy to the system rather than dissipating energy, and one would expect that this could cause the system to be unstable. If the 

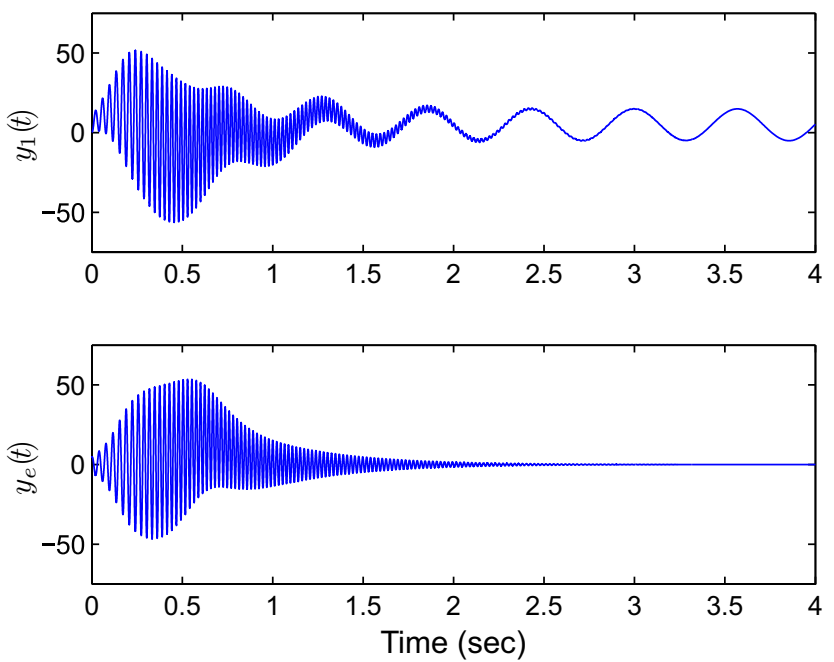

Figure 8. The output $y_{1}(t)$ asymptotically tracks the reference $y_{\mathrm{r}}(t)$, so $y_{e}(t)$ converges to zero.
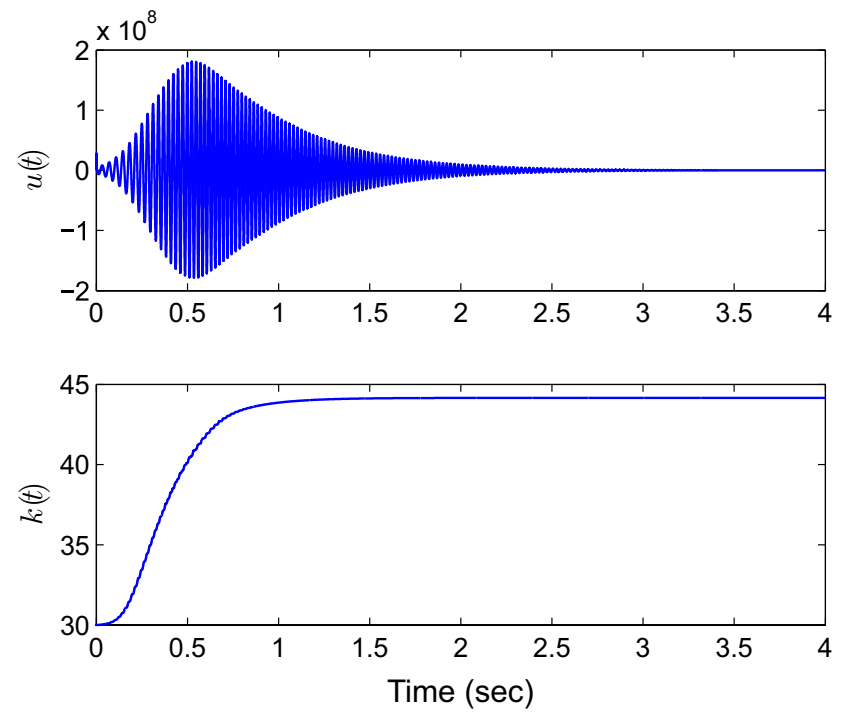

Figure 9. The control $u(t)$ is bounded and the gain $k(t)$ converges to approximately 44.2.

sensor is placed on the $m_{2}$ mass, the force-to-position system is

$$
y_{1}=G_{3}(s)(u+w)
$$

where

$$
G_{3}(s) \triangleq \frac{4 s^{3}+24 s^{2}+48 s+32}{s^{6}+12 s^{5}+36 s^{4}+72 s^{3}+74 s^{2}+128 s+100}
$$


As expected, the open-loop system (55) and (56) is unstable with unstable poles at $0.4032 \pm$ $J$ 1.3756. The mass-spring-damper system (55) and (56) is simulated with the initial conditions $q(0)=\left[\begin{array}{lll}-0.5 & 0.25 & 1.0\end{array}\right]^{\mathrm{T}} \mathrm{m}$ and $\dot{q}(0)=\left[\begin{array}{lll}0.1 & -0.2 & 0.3\end{array}\right]^{\mathrm{T}} \mathrm{m} / \mathrm{s}$. The adaptive controller $(25)$ and (44)-(48) is implemented in the feedback loop with $y_{e}(t)=y_{\mathrm{r}}(t)-y_{1}(t)$ and initial conditions $\hat{x}(0)=0$ and $k(0)=30$. Figures 8 and 9 show that $y_{e}(t)$ converges to zero and $k(t)$ converges to approximately 44.2 .

\section{CONCLUSIONS}

In this paper, we presented a direct adaptive tracking and disturbance rejection algorithm for single-input, single-output minimum-phase linear time-invariant systems with unknown-butbounded relative degree. The controller requires knowledge of the sign of the high-frequency gain and an upper bound on the magnitude of the high-frequency gain. The adaptive controller guarantees that the tracking error converges to zero. Unlike model reference adaptive control methods, this control does not require knowledge of the system order and requires only an upper bound on the relative degree. Furthermore, the adaptive controller presented herein has only one adaptive parameter regardless of system order.

In practical applications, persistent output disturbances can cause the adaptive parameter to grow without bound. This problem can be corrected for in practice by turning off the adaptation mechanism once the control objective is achieved. The adaptive controller presented in this paper is applicable to command following and disturbance rejection for steps and sinusoids with known frequency, which encompasses many practical applications. In particular, the controller has potential application to problems in structural vibration control where the disturbances are harmonic with known frequencies.

Future research may include extending this method to address the general unsolved problem of adaptive command following and disturbance rejection where the command and disturbance signals have unknown spectra. More specifically, one could consider commands and disturbances that are generated by unknown linear systems or a more general class of command and disturbances with unknown spectra, for example, broadband disturbances.

\section{APPENDIX A: PRELIMINARY RESULTS FOR ANALYSING PARAMETER-MONOTONIC ADAPTIVE SYSTEMS}

In this appendix, we present several preliminary results useful for analysing parametermonotonic adaptive systems. In this section, we consider the system

$$
\begin{aligned}
& \dot{x}=A(k) x \\
& y=C(k) x
\end{aligned}
$$

where $A(k) \in \mathbb{R}^{l \times l}$ and $C(k) \in \mathbb{R}^{d \times l}$ have entries that are polynomials in $k$.

The first two results concern the solution to a Lyapunov equation for system (A1)-(A2).

\section{Lemma A.1}

Assume that there exists $k_{\mathrm{s}}>0$ such that, for all $k \geqslant k_{\mathrm{s}}, A(k)$ is asymptotically stable. Let $Q(k) \in \mathbb{R}^{l \times l}$ have entries that are polynomial functions of $k$, where, for all $k \geqslant k_{\mathrm{s}}, Q(k)$ is positive definite. Then there exists $P:\left[k_{\mathrm{s}}, \infty\right) \rightarrow \mathbb{R}^{l \times l}$ such that each entry of $P$ is a real rational function, 
and for all $k \geqslant k_{\mathrm{s}}, P(k)$ is positive definite and satisfies

$$
A^{\mathrm{T}}(k) P(k)+P(k) A(k)=-Q(k)
$$

Proof

For all $k \geqslant k_{\mathrm{s}}$, (A3) has the unique solution

$$
P(k) \triangleq-\operatorname{vec}^{-1}\left[\left(A^{\mathrm{T}}(k) \oplus A^{\mathrm{T}}(k)\right)^{-1} \operatorname{vec} Q(k)\right]=\int_{0}^{\infty} \mathrm{e}^{A^{\mathrm{T}}(k) \tau} Q(k) \mathrm{e}^{A(k) \tau} \mathrm{d} \tau
$$

where vec is the column stacking operator and $\oplus$ is the Kronecker sum [21, Proposition 11.8.3]. Then $P(k)$ has entries that are real rational functions of $k$, and for all $k \geqslant k_{\mathrm{s}}, P(k)$ is positive definite.

\section{Lemma A.2}

Consider systems (A1)-(A2), and assume that

$$
\begin{aligned}
& A(k) \triangleq\left[\begin{array}{cc}
A_{1}(k) & A_{3}(k) \\
0 & A_{2}
\end{array}\right] \\
& C(k) \triangleq\left[C_{1}(k) C_{2}(k)\right]
\end{aligned}
$$

where $A_{1}(k) \in \mathbb{R}^{l_{1} \times l_{1}}, A_{3}(k) \in \mathbb{R}^{l_{1} \times l_{2}}, \quad C_{1}(k) \in \mathbb{R}^{d \times l_{1}}$, and $C_{2}(k) \in \mathbb{R}^{d \times l_{2}}$ have entries that are polynomials in $k$, and $A_{2} \in \mathbb{R}^{l_{2} \times l_{2}}$. For all $\lambda \in \operatorname{spec}\left(A_{2}\right)$, assume that $\lambda$ is semisimple and $\operatorname{Re} \lambda=0$. Furthermore, assume that there exists $k_{\mathrm{s}}>0$ such that, for all $k \geqslant k_{\mathrm{s}}, A_{1}(k)$ is asymptotically stable and $\lim _{t \rightarrow \infty} y(t)=0$ for all initial conditions $x(0)$. Let $\gamma>0$. Then there exist $P:\left[k_{\mathrm{s}}, \infty\right) \rightarrow$ $\mathbb{R}^{l \times l}$ and $Q:\left[k_{\mathrm{s}}, \infty\right) \rightarrow \mathbb{R}^{l \times l}$ such that the entries of $P$ and $Q$ are real rational functions, and for all $k \geqslant k_{\mathrm{s}}, P(k)$ is positive definite, $Q(k)$ is positive semidefinite, and they satisfy

$$
A^{\mathrm{T}}(k) P(k)+P(k) A(k)=-Q(k)-\gamma C^{\mathrm{T}}(k) C(k)
$$

Proof

For all $k \geqslant k_{\mathrm{s}}, \operatorname{spec}\left(A_{1}(k)\right) \cap \operatorname{spec}\left(A_{2}\right)=\emptyset$. Therefore, for all $k \geqslant k_{\mathrm{s}}$, the Sylvester equation

has the unique solution

$$
A_{1}(k) S(k)-S(k) A_{2}=A_{3}(k)
$$

$$
S(k)=\operatorname{vec}^{-1}\left[\left(-A_{2}^{\mathrm{T}} \oplus A_{1}(k)\right)^{-1} \operatorname{vec} A_{3}(k)\right]
$$

where the entries of $S(k)$ are real rational functions of $k$ [21, Corollary 7.2.5]. For all $k \geqslant k_{\mathrm{s}}$, define

$$
R(k) \triangleq\left[\begin{array}{cc}
I & -S(k) \\
0 & I
\end{array}\right]
$$

and consider the change of basis

$$
\begin{gathered}
\bar{A}(k) \triangleq R^{-1}(k) A(k) R(k)=\left[\begin{array}{cc}
A_{1}(k) & -A_{1}(k) S(k)+S(k) A_{2}+A_{3}(k) \\
0 & A_{2}
\end{array}\right]=\left[\begin{array}{cc}
A_{1}(k) & 0 \\
0 & A_{2}
\end{array}\right] \\
\bar{C}(k) \triangleq C(k) R(k)=\left[\begin{array}{ll}
C_{1}(k) & C_{2}(k)-C_{1}(k) S(k)
\end{array}\right]
\end{gathered}
$$


Since $\lim _{t \rightarrow \infty} y(t)=0$, it follows that $\lim _{t \rightarrow \infty} C_{1}(k) \mathrm{e}^{A_{1}(k) t}+\left(C_{2}(k)-C_{1}(k) S(k)\right) \mathrm{e}^{A_{2} t}=0$. Since $A_{1}(k)$ is asymptotically stable, it follows that $\lim _{t \rightarrow \infty}\left(C_{2}(k)-C_{1}(k) S(k)\right) \mathrm{e}^{A_{2} t}=0$. Since every eigenvalue of $A_{2}$ is on the imaginary axis, every non-zero entry of $\left(C_{2}(k)-C_{1}(k) S(k)\right) \mathrm{e}^{A_{2} t}$ is either constant or involves sinusoids. Therefore, $\lim _{t \rightarrow \infty}\left(C_{2}(k)-C_{1}(k) S(k)\right) \mathrm{e}^{A_{2} t}=0$ implies that $C_{2}(k)-C_{1}(k) S(k)=0$. Hence, for all $k \geqslant k_{\mathrm{s}}, \bar{C}(k)=\left[C_{1}(k) 0\right]$.

Let $Q_{1}>0$ and $\gamma>0$. Since, for all $k \geqslant k_{\mathrm{s}}, A_{1}(k)$ is asymptotically stable, it follows from Lemma A. 1 that there exists $P_{1}:\left[k_{\mathrm{s}}, \infty\right) \rightarrow \mathbb{R}^{l_{1} \times l_{1}}$ such that each entry is a real rational function and, for all $k \geqslant k_{\mathrm{s}}, P_{1}(k)$ is positive definite and satisfies

$$
A_{1}^{\mathrm{T}}(k) P_{1}(k)+P_{1}(k) A_{1}(k)=-Q_{1}-\gamma C_{1}^{\mathrm{T}}(k) C_{1}(k)
$$

Since $A_{2}$ is Lyapunov stable, it follows that there exist $P_{2}>0$ and $Q_{2} \geqslant 0$ such that $A_{2}^{\mathrm{T}} P_{2}+P_{2} A_{2}=-Q_{2}$. Next, define

$$
\begin{gathered}
\bar{P}(k) \triangleq\left[\begin{array}{cc}
P_{1}(k) & 0 \\
0 & P_{2}
\end{array}\right] \\
\bar{Q} \triangleq\left[\begin{array}{cc}
Q_{1} & 0 \\
0 & Q_{2}
\end{array}\right]
\end{gathered}
$$

so that, for all $k \geqslant k_{\mathrm{s}}, \bar{P}(k)$ is positive definite, $\bar{Q}$ is positive semidefinite, and

$$
\bar{A}^{\mathrm{T}}(k) \bar{P}(k)+\bar{P}(k) \bar{A}(k)=-\bar{Q}-\gamma \bar{C}^{\mathrm{T}}(k) \bar{C}(k)
$$

Pre-multiplying (A5) by $R^{-\mathrm{T}}(k)$ and post-multiplying by $R^{-1}(k)$ yields

$$
A^{\mathrm{T}}(k) P(k)+P(k) A(k)=-Q(k)-\gamma C^{\mathrm{T}}(k) C(k)
$$

where $P(k) \triangleq R^{-\mathrm{T}}(k) \bar{P}(k) R^{-1}(k)$ and $Q(k) \triangleq R^{-\mathrm{T}}(k) \bar{Q} R^{-1}(k)$. Thus, for all $k \geqslant k_{\mathrm{s}}, \quad P(k)$ is positive definite, $Q(k)$ is positive semidefinite, and (A4) is satisfied. Since the entries of $\bar{P}(k)$ and $R(k)$ are real rational functions, the entries of $P(k)$ and $Q(k)$ are real rational functions.

The next result concerns the derivative of a positive-definite matrix whose entries are real rational functions of a single parameter. We recall the $O$-notation. Let $f: \mathbb{R} \rightarrow \mathbb{R}$ and $g: \mathbb{R} \rightarrow \mathbb{R}$ be continuous functions. Then $f(k)=O(g(k))$ as $k \rightarrow \infty$ if there exist $M>0$ and $k_{1}>0$ such that, for all $k \geqslant k_{1},|f(k)| \leqslant M|g(k)|$. For convenience, we shall omit ' $k \rightarrow \infty$ ' from the $O$-notation for the remainder of this section.

\section{Lemma A.3}

Let $k_{\mathrm{s}}>0$ and consider $P:\left[k_{\mathrm{s}}, \infty\right) \rightarrow \mathbb{R}^{l \times l}$, where each entry of $P$ is a real rational function. Assume that, for all $k \geqslant k_{\mathrm{s}}, P(k)$ is symmetric positive definite. Then, for all $\alpha>0$, there exists $k_{2} \geqslant k_{\mathrm{s}}$ such that, for all $k \geqslant k_{2}$

$$
\frac{\mathrm{d} P(k)}{\mathrm{d} k}<\alpha P(k)
$$


Proof

Write

$$
P(k)=\left[\begin{array}{ccc}
p_{11}(k) & \cdots & p_{1 l}(k) \\
\vdots & & \vdots \\
p_{l 1}(k) & \cdots & p_{l l}(k)
\end{array}\right]
$$

where, for $i, j=1, \ldots, l, p_{i j}(k)$ is the real rational function

$$
p_{i j}(k) \triangleq \frac{\varphi_{i j}(k)}{\psi_{i j}(k)}=\frac{\varphi_{i j, u_{i j}} k^{u_{i j}}+\varphi_{i j, u_{i j}-1} k^{u_{i j}-1}+\cdots+\varphi_{i j, 1} k+\varphi_{i j, 0}}{k^{v_{i j}}+\psi_{i j, v_{i j}-1} k^{v_{i j}-1}+\cdots+\psi_{i j, 1} k+\psi_{i j, 0}}
$$

where $u_{i j}$ and $v_{i j}$ are non-negative integers. Then, for all $k>0, p_{i j}(k)$ can be expressed as

$$
p_{i j}(k)=\phi_{i j} k^{\sigma_{i j}}+\frac{k^{v_{i j}} \varphi_{i j}(k)-\phi_{i j} k^{u_{i j}} \psi_{i j}(k)}{k^{v_{i j}} \psi_{i j}(k)}
$$

where $\sigma_{i j} \triangleq u_{i j}-v_{i j}$ is an integer and for convenience $\phi_{i j} \triangleq \varphi_{i j, u_{i j}}$. The term $\left(k^{v_{i j}} \varphi_{i j}(k)-\phi_{i j} k^{u_{i j}} \psi_{i j}(k)\right) /$ $k^{v_{i j}} \psi_{i j}(k)$ is a real rational function of $k$ whose denominator is a polynomial in $k$ of degree $2 v_{i j}$ and whose numerator is a polynomial in $k$ of degree at most $u_{i j}+v_{i j}-1$. Therefore,

$$
\frac{k^{v_{i j}} \varphi_{i j}(k)-\phi_{i j} k^{u_{i j}} \psi_{i j}(k)}{k^{v_{i j}} \psi_{i j}(k)}=O\left(k^{\sigma_{i j}-1}\right)
$$

and hence $p_{i j}(k)=\phi_{i j} k^{\sigma_{i j}}+O\left(k^{\sigma_{i j}-1}\right)$.

For all $k \geqslant k_{\mathrm{s}}$, the determinants of the leading principal minors of $P(k)$ are

$$
\begin{aligned}
& \Lambda_{1}(k) \triangleq p_{11}(k) \\
& =\phi_{11} k^{\sigma_{11}}+O\left(k^{\sigma_{11}-1}\right) \\
& =\Phi_{1} k^{w_{1}}+O\left(k^{w_{1}-1}\right) \\
& \Lambda_{2}(k) \triangleq\left|\begin{array}{ll}
p_{11}(k) & p_{12}(k) \\
p_{12}(k) & p_{22}(k)
\end{array}\right| \\
& =\left|\begin{array}{ll}
\phi_{11} k^{\sigma_{11}}+O\left(k^{\sigma_{11}-1}\right) & \phi_{12} k^{\sigma_{12}}+O\left(k^{\sigma_{12}-1}\right) \\
\phi_{12} k^{\sigma_{12}}+O\left(k^{\sigma_{12}-1}\right) & \phi_{22} k^{\sigma_{22}}+O\left(k^{\sigma_{22}-1}\right)
\end{array}\right| \\
& =\Phi_{2} k^{w_{2}}+O\left(k^{w_{2}-1}\right) \\
& \Lambda_{3}(k) \triangleq\left|\begin{array}{lll}
p_{11}(k) & p_{12}(k) & p_{13}(k) \\
p_{12}(k) & p_{22}(k) & p_{23}(k) \\
p_{13}(k) & p_{23}(k) & p_{33}(k)
\end{array}\right|
\end{aligned}
$$




$$
\begin{aligned}
= & \left|\begin{array}{lll}
\phi_{11} k^{\sigma_{11}}+O\left(k^{\sigma_{11}-1}\right) & \phi_{12} k^{\sigma_{12}}+O\left(k^{\sigma_{12}-1}\right) & \phi_{13} k^{\sigma_{13}}+O\left(k^{\sigma_{13}-1}\right) \\
\phi_{12} k^{\sigma_{12}}+O\left(k^{\sigma_{12}-1}\right) & \phi_{22} k^{\sigma_{22}}+O\left(k^{\sigma_{22}-1}\right) & \phi_{23} k^{\sigma_{23}}+O\left(k^{\sigma_{23}-1}\right) \\
\phi_{13} k^{\sigma_{13}}+O\left(k^{\sigma_{13}-1}\right) & \phi_{23} k^{\sigma_{23}}+O\left(k^{\sigma_{23}-1}\right) & \phi_{33} k^{\sigma_{33}}+O\left(k^{\sigma_{33}-1}\right)
\end{array}\right| \\
= & \Phi_{3} k^{w_{3}}+O\left(k^{w_{3}-1}\right) \\
& \vdots \\
\Lambda_{l}(k) \triangleq & \triangleq P(k) \mid \\
= & \Phi_{l} k^{w_{l}}+O\left(k^{w_{l}-1}\right)
\end{aligned}
$$

where, for $i=1, \ldots, l, w_{i}$ is an integer, and $\Phi_{i} \in \mathbb{R}$ depends on the constants $\phi_{11}, \ldots, \phi_{1 i}, \ldots, \phi_{i i}$. Since $P(k)$ is positive definite for all $k \geqslant k_{\mathrm{s}}$, it follows that, for all $k \geqslant k_{\mathrm{s}}$ and for all $i=1, \ldots, l$, $\Lambda_{i}(k)>0$, and thus $\Phi_{i}>0$.

Next, consider the derivative of $P(k)$ with respect to $k$. For $i, j=1, \ldots, l$

$$
\frac{\mathrm{d} p_{i j}(k)}{\mathrm{d} k}=\sigma_{i j} \phi_{i j} k^{\sigma_{i j}-1}+O\left(k^{\sigma_{i j}-2}\right)
$$

Let $\alpha>0$. Then, for $i, j=1, \ldots, l$

$$
\begin{aligned}
\alpha p_{i j}(k)-\frac{\mathrm{d} p_{i j}(k)}{\mathrm{d} k} & =\alpha\left[\phi_{i j} k^{\sigma_{i j}}+O\left(k^{\sigma_{i j}-1}\right)\right]-\left[\sigma_{i j} \phi_{i j} k^{\sigma_{i j}-1}+O\left(k^{\sigma_{i j}-2}\right)\right] \\
& =\alpha \phi_{i j} k^{\sigma_{i j}}+O\left(k^{\sigma_{i j}-1}\right)
\end{aligned}
$$

For all $k \geqslant k_{\mathrm{s}}$, the determinants of the leading principal minors of $\alpha P(k)-\mathrm{d} P(k) / \mathrm{d} k$ are

$$
\begin{aligned}
\bar{\Lambda}_{1}(k) & \triangleq \alpha p_{11}(k)-\frac{\mathrm{d} p_{11}(k)}{\mathrm{d} k} \\
& =\alpha \phi_{11} k^{\sigma_{11}}+O\left(k^{\sigma_{11}-1}\right) \\
& =\alpha \Phi_{1} k^{w_{1}}+O\left(k^{w_{1}-1}\right) \\
\bar{\Lambda}_{2}(k) & \triangleq\left|\begin{array}{ll}
\alpha p_{11}(k)-\frac{\mathrm{d} p_{11}(k)}{\mathrm{d} k} & \alpha p_{12}(k)-\frac{\mathrm{d} p_{12}(k)}{\mathrm{d} k} \\
\alpha p_{12}(k)-\frac{\mathrm{d} p_{12}(k)}{\mathrm{d} k} & \alpha p_{22}(k)-\frac{\mathrm{d} p_{22}(k)}{\mathrm{d} k}
\end{array}\right| \\
& \left.=\mid \begin{array}{ll}
\phi_{11} k^{\sigma_{11}}+O\left(k^{\sigma_{11}-1}\right) & \phi_{12} k^{\sigma_{12}}+O\left(k^{\sigma_{12}-1}\right) \\
\phi_{12} k^{\sigma_{12}}+O\left(k^{\sigma_{12}-1}\right) & \phi_{22} k^{\sigma_{22}}+O\left(k^{\sigma_{22}-1}\right)
\end{array}\right] \mid \\
& =\alpha^{2} \Phi_{2} k^{w_{2}}+O\left(k^{w_{2}-1}\right)
\end{aligned}
$$




$$
\begin{aligned}
& \bar{\Lambda}_{3}(k) \triangleq\left|\begin{array}{ccc}
\alpha p_{11}(k)-\frac{\mathrm{d} p_{11}(k)}{\mathrm{d} k} & \alpha p_{12}(k)-\frac{\mathrm{d} p_{12}(k)}{\mathrm{d} k} & \alpha p_{13}(k)-\frac{\mathrm{d} p_{13}(k)}{\mathrm{d} k} \\
\alpha p_{12}(k)-\frac{\mathrm{d} p_{12}(k)}{\mathrm{d} k} & \alpha p_{22}(k)-\frac{\mathrm{d} p_{22}(k)}{\mathrm{d} k} & \alpha p_{23}(k)-\frac{\mathrm{d} p_{23}(k)}{\mathrm{d} k} \\
\alpha p_{13}(k)-\frac{\mathrm{d} p_{13}(k)}{\mathrm{d} k} & \alpha p_{23}(k)-\frac{\mathrm{d} p_{23}(k)}{\mathrm{d} k} & \alpha p_{33}(k)-\frac{\mathrm{d} p_{33}(k)}{\mathrm{d} k}
\end{array}\right| \\
& =\left|\alpha\left[\begin{array}{lll}
\phi_{11} k^{\sigma_{11}}+O\left(k^{\sigma_{11}-1}\right) & \phi_{12} k^{\sigma_{12}}+O\left(k^{\sigma_{12}-1}\right) & \phi_{13} k^{\sigma_{13}}+O\left(k^{\sigma_{13}-1}\right) \\
\phi_{12} k^{\sigma_{12}}+O\left(k^{\sigma_{12}-1}\right) & \phi_{22} k^{\sigma_{22}}+O\left(k^{\sigma_{22}-1}\right) & \phi_{23} k^{\sigma_{23}}+O\left(k^{\sigma_{23}-1}\right) \\
\phi_{13} k^{\sigma_{13}}+O\left(k^{\sigma_{13}-1}\right) & \phi_{23} k^{\sigma_{23}}+O\left(k^{\sigma_{23}-1}\right) & \phi_{33} k^{\sigma_{33}}+O\left(k^{\sigma_{33}-1}\right)
\end{array}\right]\right| \\
& =\alpha^{3} \Phi_{3} k^{w_{3}}+O\left(k^{w_{3}-1}\right) \\
& \bar{\Lambda}_{l}(k) \triangleq\left|\alpha P(k)-\frac{\mathrm{d} P(k)}{\mathrm{d} k}\right| \\
& =\alpha^{l} \Phi_{l} k^{w_{l}}+O\left(k^{w_{l}-1}\right)
\end{aligned}
$$

Since $\alpha>0$ and $\Phi_{1}, \ldots, \Phi_{l}>0$, it follows that, for $i=1, \ldots, l, \alpha^{i} \Phi_{i}>0$. Therefore, for all sufficiently large $k, \bar{\Lambda}_{1}(k), \ldots, \bar{\Lambda}_{l}(k)>0$, and thus $\alpha P(k)-\mathrm{d} P(k) / \mathrm{d} k$ is positive definite.

\section{APPENDIX B: PRELIMINARY RESULT FOR FORCED LINEAR TIME-VARYING SYSTEMS}

The result of this section concerns the solution to a class of forced linear time-varying systems. This result is integral to the proof of asymptotic command following and disturbance rejection for the adaptive controller presented in this paper.

\section{Lemma B.1}

Consider the nonhomogeneous linear time-varying system

$$
\dot{\zeta}(t)=A_{\mathrm{s}} \zeta(t)+\Delta(t) \zeta(t)+L \phi(t)+D \omega(t)
$$

where $\zeta \in \mathbb{R}^{l_{\zeta}}, \phi:[0, \infty) \rightarrow \mathbb{R}^{l_{\phi}}, \omega:[0, \infty) \rightarrow \mathbb{R}^{l_{\omega}}$, and $\Delta:[0, \infty) \rightarrow \mathbb{R}^{l_{\zeta} \times l_{\zeta}}$. Assume that $A_{\mathrm{s}}$ is asymptotically stable, $\Delta(\cdot)$ is continuous, $\lim _{t \rightarrow \infty} \Delta(t)=0, \phi(\cdot)$ is square integrable on $[0, \infty)$, and $\omega(\cdot)$ is bounded on $[0, \infty)$. Then, for all $\zeta(0), \zeta(\cdot)$ is bounded on $[0, \infty)$.

\section{Proof}

First, consider the system

$$
\dot{\zeta}_{1}(t)=A_{\mathrm{s}} \zeta_{1}(t)+\Delta(t) \zeta_{1}(t)+L \phi(t)
$$

Since $\Delta(\cdot)$ is continuous the solution $\zeta_{1}(\cdot)$ exists and is unique on all finite intervals. Since $A_{\mathrm{s}}$ is asymptotically stable, let $P>0$ be the solution to the Lyapunov equation $A_{\mathrm{s}}^{\mathrm{T}} P+P A_{\mathrm{s}}=-2 I$, 
and define $V\left(\zeta_{1}\right) \triangleq \zeta_{1}^{\mathrm{T}} P \zeta_{1}$. Differentiating $V\left(\zeta_{1}\right)$ along the trajectory of (B2) yields

$$
\dot{V}\left(\zeta_{1}\right)=-2 \zeta_{1}^{\mathrm{T}} \zeta_{1}+\zeta_{1}^{\mathrm{T}}\left[\Delta^{\mathrm{T}}(t) P+P \Delta(t)\right] \zeta_{1}+\zeta_{1}^{\mathrm{T}} P L \phi+\phi^{\mathrm{T}} L^{\mathrm{T}} P \zeta_{1}
$$

Since $0 \leqslant\left(\zeta_{1}-P L \phi\right)^{\mathrm{T}}\left(\zeta_{1}-P L \phi\right)$, it follows that

$$
\zeta_{1}^{\mathrm{T}} P L \phi+\phi^{\mathrm{T}} L^{\mathrm{T}} P \zeta_{1} \leqslant \zeta_{1}^{\mathrm{T}} \zeta_{1}+\phi^{\mathrm{T}} L^{\mathrm{T}} P^{2} L \phi
$$

Combining (B3) and (B4) yields

$$
\dot{V}\left(\zeta_{1}\right) \leqslant-\zeta_{1}^{\mathrm{T}} \zeta_{1}+\zeta_{1}^{\mathrm{T}}\left[\Delta^{\mathrm{T}}(t) P+P \Delta(t)\right] \zeta_{1}+\eta \phi^{\mathrm{T}} \phi
$$

where $\eta \triangleq \lambda_{\max }\left(L^{\mathrm{T}} P^{2} L\right)$. Since $\Delta(t) \rightarrow 0$ as $t \rightarrow \infty$, there exists $t_{0} \geqslant 0$ such that, for all $t \geqslant t_{0}$, $\lambda_{\max }\left(\Delta^{\mathrm{T}}(t) P+P \Delta(t)\right) \leqslant \frac{1}{2}$. Therefore, for all $t \geqslant t_{0}$

$$
\dot{V}\left(\zeta_{1}(t)\right) \leqslant-\frac{1}{2}\left\|\zeta_{1}(t)\right\|^{2}+\eta\|\phi(t)\|^{2}
$$

where $\|\cdot\|$ is the Euclidean norm. Integrating from $t_{0}$ to $t$ yields

$$
0 \leqslant V\left(\zeta_{1}(t)\right) \leqslant-\frac{1}{2} \int_{t_{0}}^{t}\left\|\zeta_{1}(\tau)\right\|^{2} \mathrm{~d} \tau+\eta \int_{t_{0}}^{t}\|\phi(\tau)\|^{2} \mathrm{~d} \tau+V\left(\zeta_{1}\left(t_{0}\right)\right)
$$

Since $\eta>0$ and $\phi(\cdot)$ is square integrable on $[0, \infty)$, it follows from (B5) that $\zeta_{1}(\cdot)$ is square integrable on $[0, \infty)$ and $V(\cdot)$ is bounded on $[0, \infty)$. Since $V(\cdot)$ is bounded on $[0, \infty), \zeta_{1}(\cdot)$ is bounded on $[0, \infty)$.

Next, consider the system

$$
\dot{\zeta}_{2}(t)=A_{\mathrm{s}} \zeta_{2}(t)+\Delta(t) \zeta_{2}(t)+D \omega(t)
$$

Since $\Delta(\cdot)$ is continuous, the solution $\zeta_{2}(\cdot)$ exists and is unique on all finite intervals. Furthermore, since $A_{\mathrm{S}}$ is asymptotically stable, there exist $\alpha>0$ and $\beta>0$ such that $\left\|\mathrm{e}^{A_{\mathrm{s}}(t-\tau)}\right\|$ $\leqslant \beta \mathrm{e}^{-\alpha(t-\tau)}$. Let $\varepsilon \in(0, \alpha)$. Since $\Delta(t) \rightarrow 0$ as $t \rightarrow \infty$, there exist $t_{0} \geqslant 0$ such that, for all $t \geqslant t_{0}$, $\|\Delta(t)\| \leqslant(\alpha-\varepsilon) / \beta$. For all $t \geqslant t_{0}$, (B6) has the solution

$$
\zeta_{2}(t)=\mathrm{e}^{A_{\mathrm{s}}\left(t-t_{0}\right)} \zeta_{2}\left(t_{0}\right)+\int_{t_{0}}^{t} \mathrm{e}^{A_{\mathrm{s}}(t-\tau)} D \omega(\tau) \mathrm{d} \tau+\int_{t_{0}}^{t} \mathrm{e}^{A_{\mathrm{s}}(t-\tau)} \Delta(\tau) \zeta_{2}(\tau) \mathrm{d} \tau
$$

which implies that

$$
\begin{aligned}
\left\|\zeta_{2}(t)\right\| \leqslant & \beta \mathrm{e}^{-\alpha\left(t-t_{0}\right)}\left\|\zeta_{2}\left(t_{0}\right)\right\|+\beta \int_{t_{0}}^{t} \mathrm{e}^{-\alpha(t-\tau)}\|D\|\|\omega(\tau)\| \mathrm{d} \tau \\
& +\beta \int_{t_{0}}^{t} \mathrm{e}^{-\alpha(t-\tau)}\|\Delta(\tau)\|\left\|\zeta_{2}(\tau)\right\| \mathrm{d} \tau
\end{aligned}
$$




$$
\begin{aligned}
\leqslant & \beta \mathrm{e}^{-\alpha\left(t-t_{0}\right)}\left\|\zeta_{2}\left(t_{0}\right)\right\|+\beta\|D\|\left(\sup _{t \geqslant t_{0}}\|\omega(t)\|\right) \int_{t_{0}}^{t} \mathrm{e}^{-\alpha(t-\tau)} \mathrm{d} \tau \\
& +\beta \int_{t_{0}}^{t} \mathrm{e}^{-\alpha(t-\tau)}\left(\frac{\alpha-\varepsilon}{\beta}\right)\left\|\zeta_{2}(\tau)\right\| \mathrm{d} \tau \\
= & \beta \mathrm{e}^{-\alpha\left(t-t_{0}\right)}\left\|\zeta_{2}\left(t_{0}\right)\right\|+\beta\|D\|\left(\sup _{t \geqslant t_{0}}\|\omega(t)\|\right)\left[\frac{1}{\alpha}-\frac{\mathrm{e}^{-\alpha\left(t-t_{0}\right)}}{\alpha}\right] \\
& +(\alpha-\varepsilon) \int_{t_{0}}^{t} \mathrm{e}^{-\alpha(t-\tau)}\left\|\zeta_{2}(\tau)\right\| \mathrm{d} \tau \\
\leqslant & c_{1}+c_{2} \mathrm{e}^{-\alpha t}+(\alpha-\varepsilon) \int_{t_{0}}^{t} \mathrm{e}^{-\alpha(t-\tau)}\left\|\zeta_{2}(\tau)\right\| \mathrm{d} \tau
\end{aligned}
$$

where $c_{1} \triangleq(1 / \alpha) \beta\|D\|\left(\sup _{t \geqslant t_{0}}\|\omega(t)\|\right)$ and $c_{2} \triangleq \beta\left\|\zeta_{2}\left(t_{0}\right)\right\| \mathrm{e}^{\alpha t_{0}}$. Multiplying (B7) by $\mathrm{e}^{\alpha t}$ yields

$$
\left\|\zeta_{2}(t)\right\| \mathrm{e}^{\alpha t} \leqslant c_{1} \mathrm{e}^{\alpha t}+c_{2}+(\alpha-\varepsilon) \int_{t_{0}}^{t}\left\|\zeta_{2}(\tau)\right\| \mathrm{e}^{\alpha \tau} \mathrm{d} \tau
$$

Using the Gronwall-Bellman inequality [2], (B8) implies that, for all $t \geqslant t_{0}$

$$
\begin{aligned}
\left\|\zeta_{2}(t)\right\| \mathrm{e}^{\alpha t} & \leqslant c_{1} \mathrm{e}^{\alpha t}+c_{2}+\int_{t_{0}}^{t}(\alpha-\varepsilon)\left(c_{1} \mathrm{e}^{\alpha \tau}+c_{2}\right) \exp \left[\int_{\tau}^{t}(\alpha-\varepsilon) \mathrm{d} s\right] \mathrm{d} \tau \\
& =c_{1} \mathrm{e}^{\alpha t}+c_{2}+(\alpha-\varepsilon) \int_{t_{0}}^{t}\left(c_{1} \mathrm{e}^{\alpha \tau}+c_{2}\right) \mathrm{e}^{(\alpha-\varepsilon)(t-\tau)} \mathrm{d} \tau \\
& =c_{1} \mathrm{e}^{\alpha t}+c_{2}+(\alpha-\varepsilon) \mathrm{e}^{(\alpha-\varepsilon) t} \int_{t_{0}}^{t} c_{1} \mathrm{e}^{\varepsilon \tau}+c_{2} \mathrm{e}^{-(\alpha-\varepsilon) \tau} \mathrm{d} \tau \\
& =c_{1} \mathrm{e}^{\alpha t}+c_{2}+(\alpha-\varepsilon) \mathrm{e}^{(\alpha-\varepsilon) t}\left[\frac{c_{1}}{\varepsilon} \mathrm{e}^{\varepsilon t}-\frac{c_{2}}{\alpha-\varepsilon} \mathrm{e}^{-(\alpha-\varepsilon) t}-\frac{c_{1}}{\varepsilon} \mathrm{e}^{\varepsilon t_{0}}+\frac{c_{2}}{\alpha-\varepsilon} \mathrm{e}^{-(\alpha-\varepsilon) t_{0}}\right] \\
& \leqslant\left[c_{1}+\frac{c_{1}(\alpha-\varepsilon)}{\varepsilon}\right] \mathrm{e}^{\alpha t}+(\alpha-\varepsilon) \mathrm{e}^{(\alpha-\varepsilon) t}\left[-\frac{c_{1}}{\varepsilon} \mathrm{e}^{\varepsilon t_{0}}+\frac{c_{2}}{\alpha-\varepsilon} \mathrm{e}^{-(\alpha-\varepsilon) t_{0}}\right] \\
& \leqslant c_{3} \mathrm{e}^{\alpha t}+c_{4} \mathrm{e}^{(\alpha-\varepsilon) t}
\end{aligned}
$$

where $c_{3} \triangleq\left[c_{1}+c_{1}(\alpha-\varepsilon) / \varepsilon\right]$ and $c_{4} \triangleq c_{2} \mathrm{e}^{-(\alpha-\varepsilon) t_{0}}$. Therefore, for all $t \geqslant t_{0}$

$$
\left\|\zeta_{2}(t)\right\| \leqslant c_{3}+c_{4} \mathrm{e}^{-\varepsilon t}
$$

and we conclude that $\zeta_{2}(\cdot)$ is bounded on $[0, \infty)$.

Now, by the superposition of the differential equations (B2) and (B6), we obtain the differential equation (B1) whose solution is given by $\zeta(t) \triangleq \zeta_{1}(t)+\zeta_{2}(t)$. Since $\zeta_{1}(\cdot)$ and $\zeta_{2}(\cdot)$ are bounded on $[0, \infty)$, it follows that $\zeta(\cdot)$ is bounded on $[0, \infty)$.

\section{REFERENCES}

1. Åström KJ, Wittenmark B. Adaptive Control (2nd edn). Addison-Wesley: Reading, MA, 1995.

2. Ioannou P, Sun J. Robust Adaptive Control. Prentice-Hall: Englewood Cliffs, NJ, 1996. 
3. Tao G. Adaptive Control Design and Analysis. Wiley: Hoboken, NJ, 2003.

4. Fradkov AL. Synthesis of an adaptive system for linear plant stabilization. Automation and Remote Control 1974; 35:1960-1966.

5. Egardt B. Stability analysis of continuous-time adaptive control. SIAM Journal on Control and Optimization 1980; 18:540-558.

6. Byrnes CI, Willems JC. Adaptive stabilization of multivariable linear systems. Proceedings of the Conference on Decision and Control, Las Vegas, NV, 1984; 1574-1577.

7. Willems J, Byrnes CI. Global Adaptive Stabilization in the Absence of Information on the Sign of the High Frequency Gain. Lecture Notes on Control and Information Sciences, vol. 62. 1984; 49-57.

8. Mareels I. A simple selftuning controller for stably invertible systems. Systems and Control Letters 1984; 4:5-16.

9. Ilchmann A. Non-Identifier-Based High-Gain Adaptive Control. Springer: Berlin, 1993.

10. Kaufman H, Barkana I, Sobel K. Direct Adaptive Control Algorithms: Theory and Applications (2nd edn). Springer: New York, 1998.

11. Polderman JW, Mareels IMY. High gain adaptive control revisited: first and second order case. Proceedings of the Conference on Decision and Control, Phoenix, AZ, 1999; 3329-3333.

12. Ilchmann A, Ryan EP. On gain adaptation in adaptive control. IEEE Transactions on Automatic Control 2003; 48:895-899.

13. Hoagg JB, Bernstein DS. Direct adaptive dynamic compensation for minimum phase systems with unknown relative degree. Proceedings of the Conference on Decision and Control, Paradise Island, The Bahamas, 2004; 183-188.

14. Hoagg JB, Bernstein DS. Direct adaptive dynamic compensation for minimum phase systems with unknown relative degree. IEEE Transactions on Automatic Control 2007, in press.

15. Xudong Y. Universal $\lambda$-tracking for nonlinearly-perturbed systems without restrictions on the relative degree. Automatica 1999; 35:109-119.

16. Bullinger E, Allgöwer F. Adaptive $\lambda$-tracking for nonlinear higher relative degree systems. Automatica 2005; 41:1191-1200.

17. Morse AS. A 4(n+1)-dimensional model reference adaptive stabilizer for any relative degree one or two, minimum phase system of dimension $n$ or less. Automatica $1987 ; \mathbf{2 3}: 123-125$.

18. Tao G, Ioannou PA. Model reference adaptive control for plants with unknown relative degree. Proceedings of the American Control Conference, Pittsburgh, PA, 1989; 2297-2302.

19. Rugh WJ. Linear Systems Theory (2nd edn). Prentice-Hall: New York, 1996.

20. Hoagg JB, Chandrasekar J, Bernstein DS. On the zeros, initial undershoot, and relative degree of collinear lumpedparameter structures. Journal of Dynamic Systems, Measurement and Control 2007, in press.

21. Bernstein DS. Matrix Mathematics. Princeton University Press: Princeton, 2005. 